\title{
1 Common Mechanism of SARS-CoV and SARS-CoV-2 Pathogenesis across Species
}

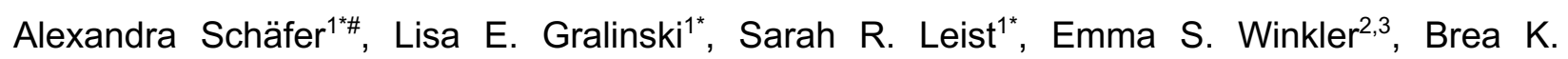
Hampton ${ }^{4,5}$, Michael A. Mooney ${ }^{6,7,8}$, Kara L. Jensen ${ }^{1}$, Rachel L. Graham ${ }^{1}$, Sudhakar Agnihothram $^{1}$, Sophia Jeng ${ }^{6,9}$, Steven Chamberlin ${ }^{7,8}$, Timothy A. Bell ${ }^{4}$, D. Trevor Scobey ${ }^{1}$, Laura A. VanBlargan², Larissa B. Thackray², Pablo Hock', Darla R. Miller ${ }^{4,10}$, Ginger D. Shaw ${ }^{4,10}$, Fernando Pardo Manuel de Villena ${ }^{4}$, Shannon K. McWeeney ${ }^{6,7,8,9}$, Stephanie A. Montgomery ${ }^{10,11}$, Michael S. Diamond ${ }^{2,3,12}$, Mark T. Heise ${ }^{4,13,17}$, Vineet D. Menachery ${ }^{14,15,16}$, Martin T. Ferris ${ }^{4 \# \text {, }}$ Ralph S. Baric ${ }^{1,13,17 \#}$

${ }^{1}$ Department of Epidemiology, University of North Carolina at Chapel Hill, Chapel Hill, NC, USA

${ }^{2}$ Department of Medicine, Washington University School of Medicine, St. Louis, MO, USA

${ }^{3}$ Department of Pathology \& Immunology, Washington University School of Medicine, St. Louis, MO, USA

${ }^{4}$ Department of Genetics, University of North Carolina at Chapel Hill, Chapel Hill, NC, USA

${ }^{5}$ Curriculum in Genetics and Molecular Biology, University of North Carolina at Chapel Hill, Chapel Hill, NC, USA

${ }^{6}$ Knight Cancer Institute, Oregon Health \& Science University, Portland, OR, USA

${ }^{7}$ Division of Bioinformatics and Computational Biology, Oregon Health \& Science University, Portland, OR, USA

${ }^{8}$ Department of Medical Informatics and Clinical Epidemiology, Oregon Health \& Science University, Portland, OR, USA

${ }^{9}$ Oregon Clinical and Translational Research Institute, Oregon Health \& Science University, Portland, OR, USA

${ }^{10}$ Lineberger Comprehensive Cancer Center, University of North Carolina at Chapel Hill, Chapel Hill, NC, USA

${ }^{11}$ Department of Pathology and Laboratory Medicine, University of North Carolina at Chapel Hill, Chapel Hill, NC

${ }^{12}$ Department of Molecular Microbiology, Washington University School of Medicine, St. Louis, MO, USA

${ }^{13}$ Department of Microbiology and Immunology, University of North Carolina at Chapel Hill, Chapel Hill, NC, USA

${ }^{14}$ Department of Microbiology and Immunology, University of Texas Medical Branch, Galveston, TX, USA 
$35{ }^{15}$ Institute for Human Infection and Immunity, University of Texas Medical Branch, Galveston TX, 36 USA

$37{ }^{16}$ Department of Pathology and Center for Biodefense \& Emerging Infectious Diseases, University

38 of Texas Medical Branch, Galveston, TX, USA

$39{ }^{17}$ Rapidly Emerging Antiviral Drug Discovery Initiative, University of North Carolina, Chapel Hill

$40 \quad \mathrm{NC}$, USA

41

$42{ }^{*}$ Co-first authors

$43+$ Co-senior authors

\section{Abstract}

47 Sarbecovirus (CoV) infections, including Severe Acute Respiratory CoV (SARS-CoV) and SARS-

48 CoV-2, are considerable human threats. Human GWAS studies have recently identified loci 49 associated with variation in SARS-CoV-2 susceptibility. However, genetically tractable models 50 that reproduce human CoV disease outcomes are needed to mechanistically evaluate genetic 51 determinants of CoV susceptibility. We used the Collaborative Cross (CC) and human GWAS 52 datasets to elucidate host susceptibility loci that regulate CoV infections and to identify host 53 quantitative trait loci that modulate severe CoV and pan-CoV disease outcomes including a major 54 disease regulating loci including CCR9. CCR9 ablation resulted in enhanced titer, weight loss, 55 respiratory dysfunction, mortality, and inflammation, providing mechanistic support in mitigating 56 protection from severe SARS-CoV-2 pathogenesis across species. This study represents a 57 comprehensive analysis of susceptibility loci for an entire genus of human pathogens conducted, 58 identifies a large collection of susceptibility loci and candidate genes that regulate multiple aspects 59 type-specific and cross-CoV pathogenesis, and also validates the paradigm of using the CC 60 platform to identify common cross-species susceptibility loci and genes for newly emerging and 61 pre-epidemic viruses.

\section{Main Text}

64 Natural host genetic variation regulates disease severity following most viral infections, yet the 65 specific genes and loci that regulate differential disease outcomes are largely unknown across 66 susceptible species ${ }^{1,2}$. Coronaviruses (CoVs) are significant human and animal pathogens; six 67 CoVs (three human, three swine) have emerged or expanded their geographic range in the $21^{\text {st }}$ 68 century $^{3,4}$. The most impactful emergent human CoVs (SARS-CoV and SARS-CoV-2) are group 
2B coronaviruses, which likely emerged from bats to cause worldwide human epidemic or pandemic respiratory infections, leading to substantial morbidity and mortality ${ }^{5,6}$. Moreover, many

71 high-risk group 2B Sarbecoviruses (SARS-like viruses) and group 2C MERS-like bat CoVs are

72 poised for future human emergence events ${ }^{7-9}$. The Sarbecovirus subgenus is clustered into four

73 clades that include the clade I SARS-CoV and high-risk SARS-like Bat CoVs (BtCoV), clade II

74 SARS-like BtCoVs like HKU3, and clade III SARS-CoV-2 (Figure 1a) ${ }^{10}$. Sarbecoviruses vary

75 widely in their ability to cause human and animal disease ${ }^{11}$. SARS-CoV caused $\sim 8,000$ infections

76 with a $10 \%$ mortality rate, while SARS-CoV-2 has infected $\sim 132$ million, leading to $\sim 2.9$ million

77 deaths to date ${ }^{12,13}$. SARS-CoV-2 infections cause asymptomatic to life-threatening disease

78 outcomes, supporting a role for inter-host genetic control of emerging viral disease outcomes in

79 both humans and mice ${ }^{14-16}$. Thus, understanding the functions of natural host variants in genes

80 that regulate susceptibility and disease severity after diverse Sarbecovirus infections may reveal

81 common genetic loci that regulate wildtype and variant virus pathogenic outcomes across

82 species, inform threat potential, and reveal novel targets for therapeutic intervention.

84 The angiotensin-converting enzyme 2 (ACE2) receptor interacts with the Spike protein (S) 85 receptor binding domains of SARS-CoV, SARS-CoV-2 and many BtCoV Sarbecoviruses, but not 86 the clade II HKU3 strain (Figure 1b) ${ }^{17}$ As many of these strains do not produce disease in mice, 87 reverse genetics and serial passaging were used to select for SARS-CoV-MA15 (SARS-MA), 88 HKU3-MA, and SARS-CoV-2 MA10 strains that replicate efficiently and produce severe disease 89 in mice $11,18,19,20$. Mouse genetic reference populations (GRPs) have been employed as highly 90 relevant models of human disease, and coupled with systems genetics data algorithms, to identify 91 host susceptibility loci, genes, genetic networks and higher-level genetic interactions that regulate 92 phenotypic variation and disease severity ${ }^{21-23}$. Among these mouse GRPs, the Collaborative 93 Cross (CC) genetic reference population encodes over 44 million single nucleotide 94 polymorphisms (SNPs), 4 million insertions and deletions (InDels), as well as several thousand 95 novel variants (both SNPs as well as InDels) present in single strains ${ }^{24,25}$. Building from our prior 96 work, genetically mapping quantitative trait loci in incipient mice from CC strains (Pre-CC ${ }^{26}$, Table 97 1), we designed a panel of $115 \mathrm{~F} 1$ crosses between different CC strains (CC-RIX; an outbred 98 population, like humans, but reproducible (Figure 1c)) to identify loci controlling Sarbecovirus 99 pathogenesis and adaptive immune responses. We used our mouse-adapted CoV models, 100 including SARS-CoV MA15 (SARS-MA), HKU3-MA, and SARS-CoV-2 MA10, which replicate 101 efficiently and produce severe disease in mice ${ }^{11,18,19,20}$, to overcome the host-specific angiotensin102 converting enzyme 2 (ACE-2) interaction with CoV Spike protein. 


\section{Results}

105 Phenotypic distributions, genomics scans, and allele effects maps for 3 traits across the 106 CC-RIX.

107 We infected the CC-RIX population with two genetically distinct Sarbecoviruses, which included 108 the clade I SARS-MA and clade II HKU3-MA strains, respectively (Figure 1a-b) ${ }^{11,27}$. Groups of 109 CC-RIX mice were inoculated with $5 \times 10^{3}$ plaque-forming units (PFU) of SARS-MA, and viral 110 burden, clinical disease (e.g., weight loss, mortality, and respiratory function), antibody titers, and

111 immune cell infiltrates were measured at multiple timepoints post-infection (ranging from 2-32 112 days) (Figure 1d, Figure 2). A matched cohort of CC-RIX was inoculated with 1x10 PFU of 113 HKU3-MA and evaluated for mortality and weight loss through day 4 post infection (Figure 2a). 114 In both studies, virus challenge elicited an array of disease phenotypes, ranging from clinically 115 inapparent infection to lethal outcomes within the first 4 days of infection. We estimated genetic 116 contributions for many of these traits and determined that heritability for these responses was $11744.4 \%-80.9 \%$, estimates that agree with previous CC studies ${ }^{26,28-31}$. Importantly, the various CC118 RIX disease phenotypes measured in response to infection appeared relatively uncorrelated

119 (Figure 2a-c), suggesting that there are many independent genetic factors driving these 120 responses. We next conducted genetic mapping to identify both genomic regions and specific 121 founder haplotypes driving various aspects of SARS-MA and HKU3-MA disease responses. 122 Given the likely complex genetic architecture underlying these phenotypes, we identified those 123 loci surpassing community-accepted significance thresholds $(p<0.33$ ), with distinct allele effects 124 (Figure 2a-c, Table 1) ${ }^{32}$. We identified 11 distinct and high-confidence loci in our RIX population 125 affecting weight loss, mortality, titer, antibody responses or respiratory function after SARS-MA 126 infection or weight loss and mortality following HKU3-MA infection. The effect sizes of these loci 127 varied from $3-23 \%$ of the total trait variation (that is, largely moderate effect sizes), the loci were 128 located in different genomic regions with different causal founder alleles, and most loci primarily 129 impacted one or a few traits in this population (Table 1). The number of independent loci and their 130 trait-specific impacts are consistent with our earlier observations of little to no correlation between 131 disease states across the RIX lines (Figure 2a-c). Together, this analysis highlights the genetic 132 complexity driving CoV disease outcomes and immunity and also the inability of any single animal 133 model of CoV disease to fully address all aspects of the disease response.

135 To investigate the possibility of pan-sarbecovirus susceptibility loci, we evaluated whether any of 136 our SARS-MA phenotypes also were associated with the haplotypes driving HKU3-MA mortality 
137 (Figure 2d, Table 1). For these analyses, we binned CC-RIX lines based on their diplotypes at 138 these HKU3-MA diseases associated loci, then determined whether these diplotype bins provided 139 an improved fit to the relationship between SARS-MA phenotypes and the CC-RIX IDs 140 themselves. For example, was there any genetic signal at quantitative trait loci (QTL) $H r S 10$ or 141 HrS11 that was associated with differential SARS-MA disease when simplifying the underlying 142 causal model; a method we have used to find additional QTLs with small effect sizes in genetically 143 complex populations ${ }^{29}$. HrS10 was associated with enhanced SARS-MA weight loss, disease and 144 mortality at day 2 post-infection (2 dpi), whereas HrS11 had moderate associations with SARS-

145 MA lung titers at both 2 and 4 dpi (Figure 2d). These results indicate that common susceptibility 146 loci regulate disease severity across two genetically distinct Sarbecovirus clades, and that $\mathrm{HrS10}$ 147 and HrS11 are both associated with HKU3-MA-induced mortality, but likely contribute to virus148 induced disease through different mechanisms.

Disease phenotypes in parental strains CC011 and CC074 and identification of a quantitative multitrait locus on chromosome 9 in the CC011xCC074-F2.

152 Concurrent with our large CC-RIX screen, we identified a pair of inbred CC strains showing highly 153 divergent susceptibilities to SARS-CoV: the disease resistant CC011/Unc and the highly 154 susceptible CC074/Unc strain (CC011 and CC074 from here on, respectively). After challenge 155 with $1 \times 10^{4}$ PFU SARS-MA, these strains exhibited marked differences in clinical and virological 156 disease phenotypes (e.g., hemorrhage, weight loss, virus titer, mortality, circulating immune 157 cells), and all CC074 mice developed lethal disease by 4 dpi post infection (Figure 3a, left panel). 158 Relevant for the current pandemic, these parental strains showed similar severe infection 159 phenotypes during SARS-CoV-2 MA10 infection (Figure 3a, right panel, Figure S1). We 160 generated 403 F2 mice by intercrossing these strains (Figure S2) and inoculated them 161 intranasally at 9-12 weeks with $1 \times 10^{4}$ PFU of SARS-MA. These F2 mice showed an expanded 162 range of disease responses relative to their parent CC strains, including mortality, weight loss, 163 titer, respiratory function, circulating immune cell and hemorrhage phenotypes (Figure S3, Figure 164 3b). We conducted QTL mapping in these F2 mice and identified five significant QTL segregating 165 in this population (HrS24-28, Table 1), and supply information on other potential loci $(p<0.33$, 166 Table S1). Most of these loci impacted multiple aspects of the SARS-MA disease response during 167 this infection time course (for example analysis of HrS26 on chromosome 9 indicated the locus 168 contributed to mortality at 4 dpi (Odds ratio (OR) of 3.15), as well as lung hemorrhage or 169 congestion (10.2\% of population variation), airflow restriction at $2 \mathrm{dpi}(7.8 \% \mathrm{PenH})$, as well as 170 peripheral neutrophil (11.8\%) and lymphocyte (12.4\%) levels) at 4 dpi (Figure 3c, Table 1). 
171 Recently, three Genome-wide Association Study (GWAS) in humans identified a locus associated

172 with respiratory failure. This locus (encompassing genes such as SLC6A20, LZTFL1, FYCO1,

173 CXCR6, XCR1, and CCR9) is syntenous with a more proximal region of our chromosome 9

174 locus ${ }^{33-35}$. CCR9 emerged as a strong candidate based on the integration of our data with these

175 studies and the presence of nonsynonomous SNPs in CCR9 as well as synonymous mutations

176 in regulatory flanking sequences.

177

178 Identification of CCR9 as a major susceptibility allele during SARS-CoV-2 infection.

179 To better understand how our contrasting CC strains and this locus regulates SARS-CoV-2 180 disease, we inoculated CC011, CC074, C57BL/6NJ and CCR9 ${ }^{-1-}$ mice on a C57BL/6NJ 181 background with SARS-CoV-2 MA10 ${ }^{20}$. CC011 and CC074 mice infected with SARS-CoV-2 182 MA10 showed concordance in their SARS- MA disease response phenotypes, including lethality 183 in the CC074 susceptible line. The CCR $9^{-/-}$mice developed more severe clinical disease (Figure 184 4a, Figure S4a), exhibited increased virus titers (Figure 4b), weight loss, mortality and prolonged pulmonary dysfunction and severe lung pathology as measured by whole body plethysmography 186 (Figure 4c), lung hemorrhage (Figure S4b), and lung damage (Figure S4c, d and Figure S4e) 187 relative to their wild-type controls, supporting its important role in protection from disease. 188 Analysis of the cytokine profile in lungs and serum by multiplex immune-assay showed increased 189 subsets of cytokines and chemokines that are involved in promoting allergic airway inflammation, 190 including IL9, IL13, II17, CCL2, CCL3, CCL5, G-CSF, and Eotaxin either in the lung tissue, serum, 191 or both (Figure $\mathbf{4 f}$ and Figure $\mathbf{S 5 b}$ ). By flow cytometric analysis, the composition of infiltrating 192 cells into the lung tissue (Figure 4e) and bronchoalveolar (BAL) fluid (Figure S5a) of CCR9-/-

193 mice showed a significant increase of CD4 ${ }^{+} \mathrm{T}$ cells, CD8 ${ }^{+}$effector $\mathrm{T}$ cells, inflammation-promoting $194 \mathrm{CD}_{11^{+}} \mathrm{DCs}$ and eosinophils at $6 \mathrm{dpi}$, consistent with an allergic airway inflammatory response. 195 Although originally found to play an important role in chronic gut inflammation, CCR9 is mainly 196 expressed on lymphocytes, dendritic cells (DCs) and monocytes/macrophages ${ }^{36,37}$, and also 197 participates in early allergic airway inflammation including the migration and proliferation of 198 eosinophils and lymphocytes ${ }^{38}$. In addition CCR $9^{+}$DCs are implicated in regulating inflammation, 199 alloimmunity, and autoimmunity ${ }^{36}$. CCR9-- mice develop chronic inflammatory responses and $200 \mathrm{CD} 11 \mathrm{~b}^{+}$inflammatory macrophages contribute to the pathogenesis of liver fibrosis via the 201 CCR9/CCL25 axis $^{39,40}$. As inflammatory macrophages contribute significantly to increased SARS202 CoV pathogenesis in mice ${ }^{41}$, together, these data target the CCR9/CCL25 axis as a major driver 203 of SARS-CoV-2 pathogenesis across species and validate CCR9 as a driver of the human Chr3 204 susceptibility loci and mouse Chr9 susceptibility locus. 
205 A key goal in animal studies is to identify relevant models of human disease. Syntenic genome

206 regions between humans and rodents often regulate a number of infectious and chronic diseases,

207 and our analysis of HrS26 extends this important pattern ${ }^{42-44}$ to sarbecovirus infections. All told, 208 the synteny between human Chr3 and mouse Chr9, the effect sizes of the loci identified in this F2 209 (between 5-15\% of the population-wide phenotypic variation in this F2, Table 1), as well as the 210 prevalence of loci impacting multiple aspects of SARS-MA associated disease, highlight how 211 sorting of multiple susceptibility alleles into individual CC strains model unique aspects of the 212 genetic control of disease responses. Furthermore, the presence of alleles of at least six of the 213 CC founder strains segregating across these loci (and often in opposite directions: a C57BL/6J 214 allele is protective at $\mathrm{HrS26}$ and $\mathrm{HrS27}$ but exacerbating at $\mathrm{HrS28}$, Table 1) highlights the utility 215 of using genetically complex but reproducible models of disease.

Identification of major effect locus on chromosome 4 and of Trim14 as a susceptibility gene during SARS-MA and SARS-CoV-2 MA10 infection.

219 Next, we revisited a previous CC-F2 intercross, CC003/UncxCC053/Unc (named CC003 and

$220 \mathrm{CC} 053$ from here on) conducted by our group ${ }^{45}$, and utilized our refined analysis pipelines once 221 the original SARS-MA disease loci (HrS5-9) were statistically accounted for. This re-analysis 222 allowed us to identify an additional locus (HrS23 on chr4) impacting both weight loss and 223 hemorrhagic damage to the lungs as determined by gross pathology (Figure 5a, b), as well as 224 several other suggestive QTLs (Table S1). The chr4 locus also overlapped with the mortality QTL 225 HrS24 identified in CC011xCC074-F2 cross (Figure 5c). SNP variation between CC003 and $226 \mathrm{CC} 053$, as well as between CC011 and CC074 in this locus pointed to Trim14 as a likely candidate 227 gene driving these differences in SARS-CoV disease. Previous work identified Trim14 as a key 228 docking platform in the context of MAVS signaling ${ }^{46,47}$. We used CRISPR/Cas9 targeting to edit 229 Trim14 in C57BL/6J mice, create a functional knockout (Figure S6), and evaluate its role following 230 infection (Figure 5d, top panel). Trim $14^{447 / \Delta 47}$ mice inoculated with $1 \times 10^{5}$ PFU of SARS-MA had 231 a modest increase in pathogenesis relative to C57BL/6J control mice. At 3 and 4 dpi, Trim14232 deficient mice had increased weight loss, which corresponded with increases in viral titer within 233 the lung at 2 and $4 \mathrm{dpi}$. This result show that an absence of Trim14 affects viral clearance. 234 Similarly, Trim $14^{447 / \Delta 47}$ mice inoculated with SARS-CoV-2 MA10 also sustained modest increases 235 in weight loss and a delayed recovery phenotype when compared to C57BL/6 mice (Figure 5d, 236 bottom panel). However, the difference in viral titer seen at early times post SARS-MA infection 237 was not observed with SARS-CoV-2 MA10. Together, these data suggest that Trim14 has a 238 shared role in attenuating Sarbecovirus disease potential, but that this effect varies between 
239 viruses. Taken together, the data demonstrates the potential of identifying common and shared

240 QTLs among group 2B coronaviruses.

\section{Discussion}

243 Across these studies, we describe several dozen loci impacting different disease responses,

244 including several which show broad responses to all tested Sarbecoviruses. We demonstrate 245 connections to human GWAS studies, and as such these data represent a resource for future 246 comparative studies of Sarbecovirus pathogenesis between humans and animals. Our study 247 highlights the power of using animal GRPs to understand the role of host genetic variation on 248 infectious diseases, generate new models of differential disease, probe the role of individual 249 genes in disease progression, and provide mechanistic insight into the role of specific host genes 250 and viral strains in regulating pathogenesis across species. In appropriately selected large 251 population screens, highly penetrant genetic variants can be identified easily, as can their impacts 252 on specific aspects of disease outcome. In contrast, targeted mapping crosses between highly 253 discordant strains can help to identify more complex genetic interaction networks such as variants 254 that are penetrant only in the context of specific genetic backgrounds, or epistatic (gene-gene) 255 interaction networks.

257 We leveraged large-scale population mapping as well as focused intercrosses to better 258 characterize the genetic susceptibility landscape of Sarbecovirus infections in mouse models ${ }^{26,45}$ 259 and demonstrated that a large number of polymorphic loci (Figure 6) regulate the host disease 260 responses to this subgroup of coronaviruses. Moreover, in this study and others, we have 261 identified specific genes (Trim55 ${ }^{26}$, Ticam $2^{45}$, and here Trim14 and CCR9), which have naturally 262 occurring polymorphisms driving aberrant SARS-CoV disease responses. Importantly, we 263 demonstrate that $\mathrm{HrS} 10$ and HrS11 influence disease severity following both clade I SARS-MA 264 and clade II HKU3-MA infection in the CC-RIX, supporting the hypothesis that intrinsic virulence 265 properties encoded within the Sarbecoviruses are subject to similar susceptibility loci in mammals. 266 Further, the concordant susceptibility profiles of CC011, CC074, Trim14 and CCR9 deficient mice 267 with SARS-MA and SARS-CoV-2 MA10 highlight the utility of pre-emergence disease models. 268 Such findings are consistent with the discovery that group I and II human norovirus infection and 269 pathogenesis are heavily regulated by polymorphisms in fucosyltransferase 2 (FUT2) ${ }^{48}$. Rich and 270 complex datasets like the ones described here enable comparisons with human GWAS studies 271 mapping QTL after SARS-CoV-2 infection ${ }^{33-35}$. The CC platform can be used to evaluate the role 272 of these loci in mouse models. One advantage of our approach is the use of different mapping 
273 platforms, which provide opportunities to combine datasets across projects to gain a greater

274 understanding of the role of how host genetic variation modulates CoV disease responses in

275 mammals (Figure 6, Table 1). In addition, the unexplained heritability and suggestive loci (Table

276 S1) we have identified, suggests that CoV disease and immunity are complex polygenic traits,

277 with the accumulation of variants across many loci driving final disease susceptibility. Collectively,

278 these studies represent the most comprehensive analysis of susceptibility loci for an entire genus

279 of human pathogens, identify a large collection of susceptibility loci and candidate genes that

280 regulate multiple aspects type-specific and cross-CoV pathogenesis, validate a role for the CCR9-

281 CCL25 axis in regulating SARS-CoV-2 disease severity and provide a resource for community

282 wide studies.

Figure Legends

285 Figure 1. Coronavirus and host- genetic model systems: a-b. Spike phylogeny of 286 representative coronaviruses and RBD alignments SARS-CoV, HKU3, and SARS-CoV-2 with emphasize on ACE2 binding residues; c.-d. The RIX Collaborative Cross Screen: The design of CC-RIX panel and the phenotypic distribution of disease phenotypes after SARSMA and HKU3-MA infection in the CC-RIX panel

a. The Spike protein sequences of selected coronaviruses were aligned and phylogenetically compared. Coronavirus genera are grouped by classic subgroup designations (1a-b, 2a-d, 3, and 4). PECoV is designated as $1 b^{*}$ because of its distinctive grouping compared with more conserved proteins. Branches in each tree are labeled with consensus support values (in \%). Sequences were aligned using free end gaps with the Blosum62 cost matrix, and the tree was constructed using the neighbor-joining method based on the multiple sequence alignment in Geneious Prime. Numbers following the underscores in each sequence correspond to the GenBank Accession number. b. Spike receptor-binding domain (RBD) alignments were performed in Geneious using free end gaps with the Blosum62 cost matrix, and 14 ACE2-critical interacting residues are highlighted in the chart. Residues in the chart are color-coded based on conservation to the SARS-CoV residue (conservation based on Blosum62). c. The Collaborative Cross is an octo-parental genetic reference panel. The population captures $\sim 40$ million SNPs and small InDels without blind spots across the genome. Each individual strain's unique combination of haplotypes across the genome results from the independent recombinations each strain experienced in their generation. As such, each CC strain represents a unique combination of Coronavirus susceptibility alleles. d. Phenotype distribution in CC-RIX panel during SARS-MA infection. Each dot represents the mean of individual CC-RIX strains. 
Figure 2. Phenotypic distributions, genomics scans, and allele effects maps for 3 traits across the CC-RIX.

a. HrS13, SARS-CoV lung titer at $2 \mathrm{dpi}, H r S 11$, percentage survival following HKU3-MA infection, and HrS12, weight loss at 4 dpi with HKU3-MA. For all 3 panels, CC-RIX strains are sorted by ascending 2 dpi SARS-MA lung titers, showing the general lack of correlation between coronavirus disease responses. b. Genome scans showing the LOD traces, as well as significance thresholds ( $p=0.33$ (orange) and $p=0.2$ (green)) for the same traits listed above. We identified HrS13 (ch16) for SARS-CoV titer, HrS11 (chr5) and HrS10 (chr13) for HKU3-MA mortality, and HrS12 for HKU3-MA weight loss. c. Allele effects at each of these loci showing causal haplotypes for HrS13 (where 129S1/SvImJ (pink) and PWK/PhJ (red) alleles cause a reduced titer, HrS10 where CAST/EiJ (green) and PWK/PhJ (red) alleles cause increased mortality, HrS11 where a 129S1/SvImJ (pink) allele causes increased mortality, and HrS12 where a PWK/PhJ (red) allele causes decreased weight loss. d. We identified relationships between HrS10 and SARS-related weight loss and clinical disease (shown is weight loss at $5 \mathrm{dpi}$ ), as well as HrS11 and SARS-MA titer (shown here is titer at $2 \mathrm{dpi}$ ), $0=$ low response haplotype, $1=1$ copy of the high response/PWK haplotype. Each dot represents data from an individual animal.

Figure 3. Disease phenotypes in parental strains CC011 and CC074 and identification of a quantitative multitrait locus on chromosome 9 in the CC011xCC074-F2.

327 a. CC011 and CC074 were identified as parental CC strains for a F2-screen based on their 328 contrasting SARS-MA induced phenotypes. Shown are weight loss, lung viral titer, percentage 329 survival, and lung hemorrhaging following SARS-MA infection ( $n=6$ for CC011 and CC074, respectively) and SARS-CoV-2 MA10 ( $n=13$ for CC011 and $n=13$ for CC074).

b. Shown as example is the phenotypic distribution for lung hemorrhage following SARS-MA infection in the

332 CC011xCC074-F2 panel. The genomic scan shows the LOD traces and significance thresholds $333(p=0.95$ (black), $p=0.90$ (blue), and $p=0.50$ (green)). The allele effect of the lung hemorrhage 334 phenotype is broken out based on the homozygous CC011 genotype, the homozygous CC074 335 genotype, or the heterozygous genotype ( $n=234$ individual CC011xCC074-F2 mice). c. A 336 quantitative multitrait locus with major effect was identified on chr9 (74.9-124 Mb), which affected 337 mortality, weight loss, hemorrhage, lung function, and peripheral hematology. CC011/Unc has a 338 C57BL/6/PWK haplotype and CC074/Unc has an A/J haplotype in this QTL region. 

infection. Infection of CCR $9^{-/-}$mice with SARS-CoV-2 MA10 showed significant differences in weight loss (a.), viral burden in the lung (b.), lung function (c.), and cytokine/chemokine distribution (d.) in the as well as in the compositions of lung infiltrating immune cells (e.) $(n=19$

$344 \mathrm{CCR9}^{-/-}$and $\mathrm{n}=19$ C57BL/6NJ; with $\mathrm{n}=4$ for $2 \mathrm{dpi}, \mathrm{n}=7$ for $4 \mathrm{dpi}, \mathrm{n}=5$ for $6 \mathrm{dpi}$, and $\mathrm{n}=3$ for $14 \mathrm{dpi}$

345 for weight loss, viral burden, congestion score, multiplex immune-assay and lung pathology;

$346 n=8$ for lung function testing; $n=12 \mathrm{CCRg}^{-/-}$and $n=13 \mathrm{C} 57 \mathrm{BL} / 6 \mathrm{NJ}$ with $\mathrm{n}=2$ each for mock, $\mathrm{n}=5-6$

347 for $4 \mathrm{dpi}$, and $\mathrm{n}=5$ for $6 \mathrm{dpi}$ for analysis of infiltrating cells). Data were analyzed using two-way-

348 ANOVA (weight loss, lung function) and Mann-Whitney test (titer, congestion score, pathology

349 scores, FACS analysis, and cytokine/chemokine analysis; ${ }^{*} p<0.05,{ }^{* *} p<0.01$.

Figure 5. Identification of major effect locus on chromosome 4 and of Trim14 as a susceptibility gene during SARS-MA and SARS-CoV-2 MA10 infection. a. Phenotypic distribution and genomic scan for HrS23 (4dpi weight loss in CC003xCC053-F2, b. HrS23 (4dpi lung hemorrhage in CC03xCC053-F2), and c. HrS24 (overall mortality in CC011xCC074-F2. d. Infection of Trim $14^{447 / \Delta 47}$ mice with SARS-MA showed significantly more weight loss and an increase in viral load in the lung compared to C57BL/6J mice over the course of a 7-day infection. A similar trend of infection progression was observed in Trim14 $4^{447 / \Delta 47}$ mice infected with SARSCoV-2 MA10; $n=10$ Trim $14^{447 / \Delta 47}$ and $n=10$ C57BL/6J for weight loss and viral load studies with SARS-CoV-2 MA10 studies; data was analyzed via student $t$ test, ${ }^{*} p<0.05,{ }^{* *} p<0.01,{ }^{* * *} p<0.005$ ).

Figure 6. Susceptibility map for SARS-MA and HKU3-MA. Depicted are significant $(p=0.95$ and $p=0.90$ ) QTLs in the Pre-CC (striped), CC-RIX (solid), and the CC011xCC074-F2 and CC003cxCC053-F2 screens, respectively (both checkered).

\section{Table1. List of significant QTLs}

\section{Supplemental Figure Legends}

368 Figure S1. CC074 show elevated viral titer at 2 dpi. Lung tissue of SARS-CoV-2 MA10 infected $369 \operatorname{CC} 011$ ( $n=6$ for $d 2 p i$ and $n=13$ for $d 4 p i)$ and CC074 ( $n=6$ for $d 2 p i$ and $n=13$ for d4pi) was tittered by plaque assay to detect viral load on d2pi and d4pi.

372 Figure S2 Design of CC011xCC074-F2 cross. In specific cases, individual CC strains may 373 present extreme outlier phenotypes, suggesting a sorting of multiple susceptibility alleles in this 
374 strain. In such cases, a mapping intercross (e.g. an F2) between two extreme strains can reveal

375 a large number of causal loci and interaction networks.

Figure S3. Phenotypic distribution of disease phenotypes after SARS-MA infection in the CC011xCC074-F2 screen. a. Lung hemorrhaging, b. weight loss at $4 \mathrm{dpi}$, c. lung titer at $4 \mathrm{dpi}$, d. percentage survival, e. peripheral blood lymphocytes, f. peripheral blood neutrophils, and $\mathbf{g}$. PenH lung function. Each dot represents a single animal.

Figure S4. CCR9 ${ }^{-/-}$mice show significant mortality and lung pathology starting by d6pi of infection. Infection of CCR9 ${ }^{-/-}$mice with SARS-CoV-2 MA10 showed significant differences in mortality (a.), congestion score (b.), lung pathology (c. and d.). Representative H\&E stains of lung tissue sections are shown for 4, 6, and $14 \mathrm{dpi}$ for $\mathrm{CCR9^{-/ }}$ and $\mathrm{C} 57 \mathrm{BL} / 6 \mathrm{NJ}$, scale bar indicates $100 \mu \mathrm{m}$ (e.), (n=19 CCR9-/- and $n=19$ C57BL/6NJ; with $n=4$ for $2 \mathrm{dpi}, \mathrm{n}=7$ for $4 \mathrm{dpi}, \mathrm{n}=5$ for 6 dpi, and $n=3$ for 14 dpi congestion score and histopathology scoring. Data were analyzed using logrank (mortality) and Mann-Whitney test (congestion score and pathology scores), ${ }^{*} \mathrm{p}<0.05$.

Figure S5. Infiltrating cells in BAL of CCR9-/- and C57BL/6NJ mice after SARS-CoV-2 MA10 infection. Infection of CCR9-- mice with SARS-CoV-2 MA10 showed significant differences in the compositions of lung infiltrating immune cells in BAL (a.) as well as in cytokine/chemokine distribution in serum (b.) ( $n=19 \mathrm{CCR}^{-/-}$and $n=19 \mathrm{C} 57 \mathrm{BL} / 6 \mathrm{NJ}$; with $n=4$ for $2 \mathrm{dpi}, \mathrm{n}=7$ for $4 \mathrm{dpi}, \mathrm{n}=5$ for $6 \mathrm{dpi}$, and $\mathrm{n}=3$ for 14 dpi for multiplex immune-assay and $\mathrm{n}=12$ $\mathrm{CCR9}^{-{ }^{--}}$and $\mathrm{n}=13$ C57BL/6NJ with $\mathrm{n}=2$ each for mock, $\mathrm{n}=5-6$ for $4 \mathrm{dpi}$, and $\mathrm{n}=5$ for $6 \mathrm{dpi}$ for analysis of infiltrating cells). Data were analyzed using Mann-Whitney test, ${ }^{*} p<0.05,{ }^{* *} p<0.01$.

Figure S6. Lung tissue from Trim $14^{447 / 447}$ mice lacked detectable Trim14 mRNA. Tissue from Trim14 $4^{44 / \Delta 47}$ were found to lack detectable Trim14 mRNA, likely due to nonsense-mediated

Figure S7. Gating schemes for flow cytometry analysis. 
This study was supported by grants in aid from the National Institutes of Health, Allergy and Infectious Diseases (Al100625 and Al149644 to R.S.B., M.T.H., M.T.F., and F.P.M.V.), (R00AG049092 to V.D.M.), a contract from the NIH (HHSN272201700036I; Task Order \#38 75N93020F00001 to R.S.B), and support from NCATS (UL1TR002369 to M.A.M. and S.K.M.). We would like to thank the Systems Genetics Core Facility (UNC) for maintaining and distributing

412 Collaborative Cross mice. The research was also supported by a generous gift from the Chan-

413 Zuckerberg foundation.

\section{Author contributions}

A.S., L.E.G., F.P.M.V., S.K.M., M.T.H., V.D.M., M.T.F., R.S.B designed screens and biology experiments; A.S., L.E.G., S.R.L., V.D.M. conducted and characterized infections in vivo; A.S.,

418 L.E.G., S.R.L., E.S.W., K.L.J., D.T.S. performed in vitro studies and immune cell quantifications; A.S., L.E.G, S.R.L., R.L.G., S.A.M., V.D.M., M.T.F. processed and analyzed data, and generated figures; A.S., B.K.H, M.A.M., S.J., S.C., S.K.M., M.T.F. performed genetic mapping studies; L.A.V., L.B.T., M.S.D. designed and isolated Trim14-deficient mice; A.S., L.E.G., V.D.M. performed in vivo evaluation of Trim14-deficient infected animals; S.A.M. scored pathologic changes in the lungs of infected mice; R.L.G., S.A. isolated recombinant viruses; T.A.B., P.H., G.D.S., D.R.M. produced CC-RIX and F2 animals, and processed samples for genotyping; L.E.G., S.R.L., B.K.H, M.A.M., K.L.J., S.J., T.A.B., L.B.T., D.R.M., G.D.S., M.S.D., F.P.M.V., S.K.M., M.T.H. edited the manuscript; A.S., M.T.F., R.S.B. wrote the manuscript.

\section{Competing interests}

429 The authors have no competing interests.

\section{References}

4321 Ge, D. et al. Genetic variation in IL28B predicts hepatitis C treatment-induced viral clearance. Nature 461, 399-401, doi:10.1038/nature08309 (2009). genetic contribution to variation of HIV-1 virus load. Proc Natl Acad Sci U S A 112, 14658-14663, doi:10.1073/pnas.1514867112 (2015).

3 Wang, Q., Vlasova, A. N., Kenney, S. P. \& Saif, L. J. Emerging and re-emerging coronaviruses in pigs. Curr Opin Virol 34, 39-49, doi:10.1016/j.coviro.2018.12.001 (2019). Chen, B. et al. Overview of lethal human coronaviruses. Signal Transduct Target Ther 5, 4 Chen, B. et al. Overview of lethal human co origin. Nature 579, 270-273, doi:10.1038/s41586-020-2012-7 (2020). 
4446 Zhang, Y. Z. \& Holmes, E. C. A Genomic Perspective on the Origin and Emergence of SARS-CoV-2. Cell 181, 223-227, doi:10.1016/j.cell.2020.03.035 (2020).

4467 Menachery, V. D. et al. SARS-like WIV1-CoV poised for human emergence. Proc Natl Acad Sci U S A 113, 3048-3053, doi:10.1073/pnas.1517719113 (2016). Menachery, V. D. et al. A SARS-like cluster of circulating bat coronaviruses shows potential for human emergence. Nat Med 21, 1508-1513, doi:10.1038/nm.3985 (2015). Anthony, S. J. et al. Further Evidence for Bats as the Evolutionary Source of Middle East Respiratory Syndrome Coronavirus. mBio 8, doi:10.1128/mBio.00373-17 (2017).

10 Coronaviridae Study Group of the International Committee on Taxonomy of, V. The species Severe acute respiratory syndrome-related coronavirus: classifying 2019-nCoV and naming it SARS-CoV-2. Nat Microbiol 5, 536-544, doi:10.1038/s41564-020-0695-z (2020).

11 Becker, M. M. et al. Synthetic recombinant bat SARS-like coronavirus is infectious in cultured cells and in mice. Proc Natl Acad Sci U S A 105, 19944-19949, doi:10.1073/pnas.0808116105 (2008). Pandemic and Economic Impact. Pak J Med Sci 36, S73-S78, doi:10.12669/pjms.36.COVID19-S4.2638 (2020). Enserink, M. \& Kupferschmidt, K. With COVID-19, modeling takes on life and death importance. Science 367, 1414-1415, doi:10.1126/science.367.6485.1414-b (2020).

14 Rasmussen, A. L. et al. Host genetic diversity enables Ebola hemorrhagic fever pathogenesis and resistance. Science 346, 987-991, doi:10.1126/science.1259595 (2014). Sanchez, A., Wagoner, K. E. \& Rollin, P. E. Sequence-based human leukocyte antigen-B typing of patients infected with Ebola virus in Uganda in 2000: identification of alleles associated with fatal and nonfatal disease outcomes. J Infect Dis 196 Suppl 2, S329-336, doi:10.1086/520588 (2007).

16 Cameron, M. J. et al. Interferon-mediated immunopathological events are associated with atypical innate and adaptive immune responses in patients with severe acute respiratory syndrome. J Virol 81, 8692-8706, doi:10.1128/JVI.00527-07 (2007).

17 Shang, J. et al. Structure of mouse coronavirus spike protein complexed with receptor reveals mechanism for viral entry. PLoS Pathog 16, e1008392, doi:10.1371/journal.ppat.1008392 (2020).

18 Roberts, A. et al. A mouse-adapted SARS-coronavirus causes disease and mortality in BALB/c mice. PLoS Pathog 3, e5, doi:10.1371/journal.ppat.0030005 (2007).

19 Dinnon, K. H. et al. A mouse-adapted SARS-CoV-2 model for the evaluation of COVID19 medical countermeasures. bioRxiv, doi:10.1101/2020.05.06.081497 (2020). mortality in Standard Laboratory Mice. Cell, doi:10.1016/j.cell.2020.09.050 (2020).

21 Leist, S. R. \& Baric, R. S. Giving the Genes a Shuffle: Using Natural Variation to Understand Host Genetic Contributions to Viral Infections. Trends Genet 34, 777-789, doi:10.1016/j.tig.2018.07.005 (2018).

22 Schafer, A., Baric, R. S. \& Ferris, M. T. Systems approaches to Coronavirus pathogenesis. Curr Opin Virol 6, 61-69, doi:10.1016/j.coviro.2014.04.007 (2014). Resource for Studying Host-Pathogen Interactions. Cell Host Microbe 25, 484-498, 
24 Srivastava, A. et al. Genomes of the Mouse Collaborative Cross. Genetics 206, 537-556, doi:10.1534/genetics.116.198838 (2017). Collaborative Cross, $\mathrm{C}$. The genome architecture of the Collaborative Cross mouse genetic reference population. Genetics 190, 389-401, doi:10.1534/genetics.111.132639 (2012). Using the Collaborative Cross. PLoS Genet 11, e1005504, doi:10.1371/journal.pgen.1005504 (2015).

Frieman, M. et al. Molecular determinants of severe acute respiratory syndrome coronavirus pathogenesis and virulence in young and aged mouse models of human disease. J Virol 86, 884-897, doi:10.1128/JVI.05957-11 (2012). Maurizio, P. L. et al. Bayesian Diallel Analysis Reveals Mx1-Dependent and Mx1Independent Effects on Response to Influenza A Virus in Mice. G3 (Bethesda) 8, 427 -

29 Noll, K. E. et al. Complex Genetic Architecture Underlies Regulation of Influenza-AVirus-Specific Antibody Responses in the Collaborative Cross. Cell Rep 31, 107587, doi:10.1016/j.celrep.2020.107587 (2020).

30 Aylor, D. L. et al. Genetic analysis of complex traits in the emerging Collaborative Cross. Genome Res 21, 1213-1222, doi:10.1101/gr.111310.110 (2011). Abiola, O. et al. The nature and identification of quantitative trait loci: a community's view. Nat Rev Genet 4, 911-916, doi:10.1038/nrg1206 (2003).

doi:10.1038/s41586-020-03065-y (2020).

35 Shelton, J. F. et al. Trans-ancestry analysis reveals genetic and nongenetic associations with COVID-19 susceptibility and severity. Nat Genet, doi:10.1038/s41588-021-00854-7 (2021).

36 Pathak, M. \& Lal, G. The Regulatory Function of CCR9(+) Dendritic Cells in Inflammation and Autoimmunity. Front Immunol 11, 536326, doi:10.3389/fimmu.2020.536326 (2020).

37 Wang, C. et al. The role of chemokine receptor 9/chemokine ligand 25 signaling: From immune cells to cancer cells. Oncol Lett 16, 2071-2077, doi:10.3892/ol.2018.8896 (2018).

38 Lopez-Pacheco, C., Soldevila, G., Du Pont, G., Hernandez-Pando, R. \& Garcia-Zepeda, E. A. CCR9 Is a Key Regulator of Early Phases of Allergic Airway Inflammation. Mediators Inflamm 2016, 3635809, doi:10.1155/2016/3635809 (2016).

39 Nakamoto, N. Role of inflammatory macrophages and CCR9/CCL25 chemokine axis in the pathogenesis of liver injury as a therapeutic target. Nihon Rinsho Meneki Gakkai Kaishi 39, 460-467, doi:10.2177/jsci.39.460 (2016).

40 Chu, P. S. et al. C-C motif chemokine receptor 9 positive macrophages activate hepatic stellate cells and promote liver fibrosis in mice. Hepatology 58, 337-350, doi:10.1002/hep.26351 (2013). 
53541 Channappanavar, R. et al. Dysregulated Type I Interferon and Inflammatory MonocyteMacrophage Responses Cause Lethal Pneumonia in SARS-CoV-Infected Mice. Cell Host Microbe 19, 181-193, doi:10.1016/j.chom.2016.01.007 (2016).

42 Murdoch, B. M. et al. Genome-wide scan identifies loci associated with classical BSE occurrence. PLoS One 6, e26819, doi:10.1371/journal.pone.0026819 (2011).

43 Prisco, S. Z. et al. Refined mapping of a hypertension susceptibility locus on rat chromosome 12. Hypertension 64, 883-890, doi:10.1161/HYPERTENSIONAHA.114.03550 (2014).

44 Jamieson, S. E. et al. Evidence for a cluster of genes on chromosome 17q11-q21 controlling susceptibility to tuberculosis and leprosy in Brazilians. Genes Immun 5, 4657, doi:10.1038/sj.gene.6364029 (2004).

45 Gralinski, L. E. et al. Allelic Variation in the Toll-Like Receptor Adaptor Protein Ticam2 Contributes to SARS-Coronavirus Pathogenesis in Mice. G3 (Bethesda) 7, 1653-1663, doi:10.1534/g3.117.041434 (2017). Zhou, Z. et al. TRIM14 is a mitochondrial adaptor that facilitates retinoic acid-inducible gene-I-like receptor-mediated innate immune response. Proc Natl Acad Sci U S A 111, E245-254, doi:10.1073/pnas.1316941111 (2014).

47 Tan, P. et al. Assembly of the WHIP-TRIM14-PPP6C Mitochondrial Complex Promotes RIG-I-Mediated Antiviral Signaling. Mol Cell 68, 293-307 e295, doi:10.1016/j.molcel.2017.09.035 (2017).

48 Lindesmith, L. et al. Human susceptibility and resistance to Norwalk virus infection. Nat Med 9, 548-553, doi:10.1038/nm860 (2003).

49 Menachery, V. D., Gralinski, L. E., Baric, R. S. \& Ferris, M. T. New Metrics for Evaluating Viral Respiratory Pathogenesis. PLoS One 10, e0131451, doi:10.1371/journal.pone.0131451 (2015).

50 Leist, S. R., Jensen, K. L., Baric, R. S. \& Sheahan, T. P. Increasing the translation of

51 Sheahan, T. P. et al. Comparative therapeutic efficacy of remdesivir and combination lopinavir, ritonavir, and interferon beta against MERS-CoV. Nat Commun 11, 222, doi:10.1038/s41467-019-13940-6 (2020).

52 Sigmon, J. S. et al. Content and performance of the MiniMUGA genotyping array, a new tool to improve rigor and reproducibility in mouse research. bioRxiv, 2020.2003.2012.989400, doi:10.1101/2020.03.12.989400 (2020).

53 Gatti, D. M. et al. Quantitative trait locus mapping methods for diversity outbred mice. G3 (Bethesda) 4, 1623-1633, doi:10.1534/g3.114.013748 (2014).

54 Broman, K. W., Wu, H., Sen, S. \& Churchill, G. A. R/qtl: QTL mapping in experimental crosses. Bioinformatics 19, 889-890, doi:10.1093/bioinformatics/btg112 (2003).

\section{Online Material and Methods}

\section{Cells and viruses}

576 Recombinant mouse-adapted SARS-CoV MA15 (SARS-MA), HKU3-SRBD-MA (HKU3-MA), and 577 SARS-CoV-2 MA10 (SARS-2-MA) virus were generated as described previously (HKU3-SRBD578 MA: GenBank Accession Number XXX, SARS-CoV-2 MA10: GenBank Accession Number 
$X X X)^{11,18,20}$. For virus titration, the caudal lobe of the right lung was homogenized in PBS, resulting homogenate was serial-diluted and inoculated onto confluent monolayers of Vero E6 cells (ATCC $X X X)$, followed by agarose overlay. Plaques were visualized with overlay of Neutral Red dye on day 2 (SARS-MA) or day 3 (SARS-CoV-2 MA10) post infection.

\section{Mouse studies and in vivo infections}

All mouse studies were performed at the University of North Carolina (Animal Welfare Assurance \#A3410-01) using protocols approved by the UNC Institutional Animal Care and Use Committee (IACUC). Animal studies at Washington University were carried out in accordance with the recommendations in the Guide for the Care and Use of Laboratory Animals of the National Institutes of Health. The protocols were approved by the IACUC at the Washington University School of Medicine (Assurance number A3381-01).

Mouse studies fall into three major classes: CC-RIX, F2 intercross mice, and inbred wild-type or gene-edited mice. The laboratory of Pardo Manuel de Villena (FPMV) purchased CC mice from the Systems Genetics Core Facility at UNC between 2012 and 2018. These CC mice were used to breed CC-RIXs in the FPMV laboratory, to ensure proper cohorts and batch sizes. CC-RIXs were generated in a ring design such that each CC-RIX had one copy of the MHC H2B $\mathrm{B}^{\mathrm{b}}$ allele, and that each CC strain was used as both dam and sire in equal proportion across all RIXs. Mice ( 105 CC-RIX strains, 3 animals each) were transferred at 5-6 weeks of age to the Baric (RSB) laboratory for infection

between 9-12 weeks of age.

599 The details of the CC003 x CC053 F2 are published ${ }^{45}$. The Systems Genetics Core Facility was 600 contracted to generate the F2 cross between CC011 and CC074. F1 mice between CC011 and 601 CC074 were generated in both potential cross directions, and F2 mice were bred in all 4 possible 602 F1 x F1 combinations, to ensure appropriately balanced sex chromosome and parent-of-origin 603 effects. F2 mice (226 males, 177 females) were weaned such that littermates were randomized 604 to different experimental cages to further reduce litter- or batch-effects on the study, and mice 605 were transferred at 5-6 weeks of age to the RSB laboratory for infection between 9-12 weeks of 606 age.

607 15- week old CCR9 ${ }^{-/-}$mice (strain 027041) and 15-week old female C57BL/6NJ mice (strain 608 005304) were purchased from Jackson Laboratory. CC-RIX, CC-F2 mice, Trim14-deficient, and $609 \mathrm{CCR}^{-/-}$mice were infected with $5 \times 10^{3}$ (CC-RIX with SARS-MA), $1 \times 10^{4}$ (CC-F2 with SARS-MA 610 and SARS-CoV-2 MA10), and $1 \times 10^{5}$ (CC-RIX with HKU3-MA, Trim14447/A47 and CCR9-/- mice with 611 SARS-MA and SARS-CoV-2 MA10) plaque forming units (PFU) in $50 \mu$ PBS intranasally at 9-12 612 (CC-RIX and CC-F2 mice) or 15 (CCR9 ${ }^{-/}$and C57BL/6NJ) weeks of age, respectively. Body 
613 weight, mortality, and pulmonary function by whole body plethysmography ${ }^{49}$ were monitored daily

614 where indicated. At indicated timepoints, mice were euthanized and gross pathology (hemorrhage

615 score) of the lung was assessed and scored on a scale from 0 (no hemorrhage) to 4 (severe

616 hemorrhage affecting all lung lobes). Then lung tissue was harvested for titer and histopathology

617 analysis; and blood samples were harvested to determine antibody composition and for analysis

618 of peripheral immune cells. Samples were stored at $-80^{\circ} \mathrm{C}$ until homogenized and titered by

619 plaque assay as described above. Serum was prepared and SARS-CoV spike-specific antibody

620 were quantified by ELISA as previously described ${ }^{33}$. Peripheral blood was diluted 1:5 in

621 PBS/EDTA and analyzed with the VetScan HM5 as previously described ${ }^{50}$. Histopathology

622 samples were fixed in 10\% phosphate buffered formalin for 7 days before paraffin embedding,

623 sectioning stained with hematoxylin and eosin.

\section{Generation of Trim14-deficient mice}

626 Gene-edited Trim14-deficient mice were generated with support from the Genome Engineering 627 and iPSC center and Department of Pathology Micro-Injection Core (Washington University 628 School of Medicine). A sgRNA targeting exon 4 of Trim14 was selected based on minimal off629 target effects in silico and targeting efficiency in vitro. The sgRNA (5'630 ACCAATGGACACTCGCCTGANGG-3') was synthesized, transcribed (HiScribe T7 In vitro 631 Transcription Kit, New England BioLabs), and purified (MEGAclear Transcription Clean-Up Kit, 632 Thermo Fisher). The sgRNA was mixed and co-injected with Cas9 RNA at 5ng/ $\mu$ and $10 \mathrm{ng} / \mu \mathrm{l}$ 633 final concentrations into half-day-old C57BL/6J embryos (E0.5). After next-generation sequencing 634 of founders and two generations of mice backcrossed to C57BL/6J mice, a mouse line with a 47635 nucleotide deletion

636 GCCTGAAGGAAAGTGAGTTGCCTAAGACCAACTCCAAGTCCTTGCTC-3') encompassing the $6373^{\prime}$ splice site of intron 4 and part of the coding region of exon 4 was generated. These Trim14 $4^{47 / \Delta 47}$ 638 mice were bred as homozygotes and used for experiments. Trim $14^{447 / \Delta 47}$ mice were born in normal 639 Mendelian frequencies and showed no apparent defects in development, growth, or fecundity. 640 Lung tissue from Trim14 ${ }^{447 / \Delta 47}$ were found to lack detectable Trim14 mRNA, likely due to 641 nonsense-mediated decay, as measured by RT-qPCR using a predesigned primer/probe set for 642 Trim14 (IDT, Assay ID Mm.PT.58.286730) and the housekeeping gene GAPDH (IDT, Assay ID 643 Mm.PT.39a.1). Sanger sequencing of a polymerase chain reaction amplicon [5' 644 GGCACAGCTCAACCCATGG -3' (forward) and 5'- ACCAGCGAGCTCGTGCTCC -3' (reverse)] 645 was used for genotyping. 
Flow cytometry analysis of immune cell infiltrates.

648 For analysis of BAL fluid, mice were sacrificed by ketamine overdose, followed by cannulation of

649 the trachea with a 19-G canula. BAL was performed with three washes of $0.8 \mathrm{ml}$ of sterile PBS.

650 BAL fluid was centrifuged, and single cell suspensions were generated for staining. For analysis

651 of lung tissues, mice were perfused with sterile PBS, and the right inferior lung lobes were

652 digested at $37^{\circ} \mathrm{C}$ with $630 \mu \mathrm{g} / \mathrm{mL}$ collagenase $\mathrm{D}$ (Roche) and $75 \mathrm{U} / \mathrm{mL}$ of DNase I (Sigma-Aldrich)

653 for $2 \mathrm{~h}$. Single cell suspensions of BAL fluid and lung digest were preincubated with Fc Block

654 antibody (BD PharMingen) in PBS + 2\% heat-inactivated FBS for $10 \mathrm{~min}$ at room temperature

655 before staining. Cells were incubated with antibodies against the following markers: efluor506

656 Viability Dye (Thermo Fisher, 65-0866-14), BUV395 anti- CD45 (Clone 30-F11, BD Biosciences),

657 BV711 anti-CD11b (Clone M1/70, Biolegend), APC-Cy7 anti-CD11c (Clone HL3, BD

658 Biosciences), BV650 anti-Ly6G (Clone 1A8, Biolegend), Pacific Blue anti-Ly6C (Clone HK1.4,

659 Biolegend) FITC anti-CD24 (Clone M1/69, Biolegend), PE anti-Siglec F (Clone E50-2440,

660 Biolegend), PE-Cy7 anti-CD64 (Clone X54-5/7.1, Biolegend), AF700 anti-MHCII (Clone

661 M5/114.15.2, Biolegend), BV421 anti-CD3 (Clone 17A2, Biolegend), BV785 anti-CD4 (Clone

662 GK1.5, Biolegend), APC anti-CD8a (Clone 53-6.7, Biolegend) BV421 anti-B220 (Clone RA3-

663 6B2, Biolegend) APC-Cy7 anti-CD44 (Clone IM7, Biolegend) BV605 anti-CD62L (Clone MEL-14,

664 Biolegend). All antibodies were used at a dilution of 1:200. Cells were stained for 20 min at $4^{\circ} \mathrm{C}$,

665 washed, fixed and permeabilized for intracellular staining with Foxp3/Transcription Factor

666 Staining Buffer Set (eBioscience) according to manufacturer's instructions. Cells were incubated

667 overnight at $4^{\circ} \mathrm{C}$ with BV421 anti-Foxp3 (Clone MF-14, Biolegend) washed, re-fixed with 4\% PFA

668 (EMS) for $20 \mathrm{~min}$ and resuspended in permeabilization buffer. Absolute cell counts were

669 determined using Trucount beads (BD). Flow cytometric data were acquired on a cytometer (BD-

670 X20; BD Biosciences) and analyzed using FlowJo software (Tree Star) (Figure S7).

672 Cytokine and chemokine protein analysis

673 The small center lung lobe of each mouse was homogenized in $1 \mathrm{ml}$ of PBS and briefly centrifuged

674 to remove debris. Fifty microliters of homogenate were used to measure cytokine and chemokine

675 protein abundance using a Bio-Plex Pro mouse cytokine 23-plex assay (Bio-Rad) according to

676 the manufacturer's instructions.

\section{Lung pathology scoring}

679 Two separate lung pathology scoring scales, Matute-Bello and Diffuse Alveolar Damage (DAD), 680 were used to quantify acute lung injury $(A L I)^{51}$. 
681 For Matute-Bello scoring samples were blinded and three random fields of lung tissue were 682 chosen and scored for the following: (A) neutrophils in alveolar space (none = 0, 1-5 cells $=1$, > 6835 cells $=2$ ), (B) neutrophils in interstitial space (none $=0,1-5$ cells $=1,>5$ cells $=2$ ), $(C)$ hyaline 684 membranes (none $=0$, one membrane $=1,>1$ membrane $=2$ ), (D) Proteinaceous debris in air 685 spaces (none $=0$, one instance $=1,>1$ instance $=2)$, $(E)$ alveolar septal thickening $(<2 \AA \sim$ mock 686 thickness $=0,2-4 \AA \sim$ mock thickness $=1,>4 \AA \sim$ mock thickness $=2$ ). Scores from A-E were put 687 into the following formula score $=[(20 \times A)+(14 \times B)+(7 \times C)+(7 \times D)+(2 \times E)] / 100$ to obtain a 688 lung injury score per field and then averaged for the final score for that sample.

689 In a similar way, for DAD scoring, three random fields of lung tissue were scored for the in a 690 blinded manner for: $1=$ absence of cellular sloughing and necrosis, $2=$ uncommon solitary cell 691 sloughing and necrosis (1-2 foci/field), 3=multifocal (3+foci) cellular sloughing and necrosis with 692 uncommon septal wall hyalinization, or $4=$ multifocal ( $>75 \%$ of field) cellular sloughing and 693 necrosis with common and/or prominent hyaline membranes. To obtain the final DAD score per 694 mouse, the scores for the three fields per mouse were averaged.

\section{Genotyping}

CC003, CC053, their F1 progeny, and the F2 cross were genotyped as previously described ${ }^{45}$. CC011, CC074, their F1 progeny, and the F2 cross were genotyped on the MiniMUGA genotyping array $^{52}$. Genomic DNA was isolated from tail-clips of animals using the Qiagen (Hilden, Germany) DNeasy Blood \& Tissue kit. $1.5 \mu \mathrm{g}$ was sent to Neogen (Lincoln, Nebraska) for processing. We filtered the genotypes upon return for informativeness within this cross. To be considered informative, the marker had to have one homozygous allele in all CC011 mice genotyped, the alternate homozygous allele in all CC074 mice genotyped, and the appropriate call in all F1 animals ( $\mathrm{H}$ calls on the autosomes, an $\mathrm{H}$ call in females on the $\mathrm{X}$ chromosomes, and the relevant homozygous call in male F1s). This filtering reduced the $\sim 10,800$ SNPs on the MiniMUGA array to 2821 informative markers.

\section{QTL mapping and statistical analyses}

709 For the CC-RIX, we used the same pipeline we previously described ${ }^{29}$. Briefly, each CC-RIX had 710 their genome represented as an array of probabilities of each of the $8 \mathrm{CC}$ founder haplotypes

711 (Figure 1C). This array was used in the DOQTL R package ${ }^{53}$ to run an 8-allele regression at each 712 of 77,000 markers for our CC-RIX phenotypes. At each marker, a LOD score is calculated 713 describing the goodness of fit of our trait genotype model relative to a null model. Significance 714 was determined by running 1000 permutations scrambling the relationship between phenotypes 
715 and haplotypes. In this way, significance is independent of both population allele frequencies, as

716 well as the phenotypic distribution. For the F2 crosses, instead of a regression on haplotype

717 probabilities, the R/QTL package conducts a regression of the trait of interest on the exact

718 genotypes at each locus ${ }^{54}$. As with the CC-RIX mapping, permutation testing is used to identify

719 significance.

720 
Figure 1

a.

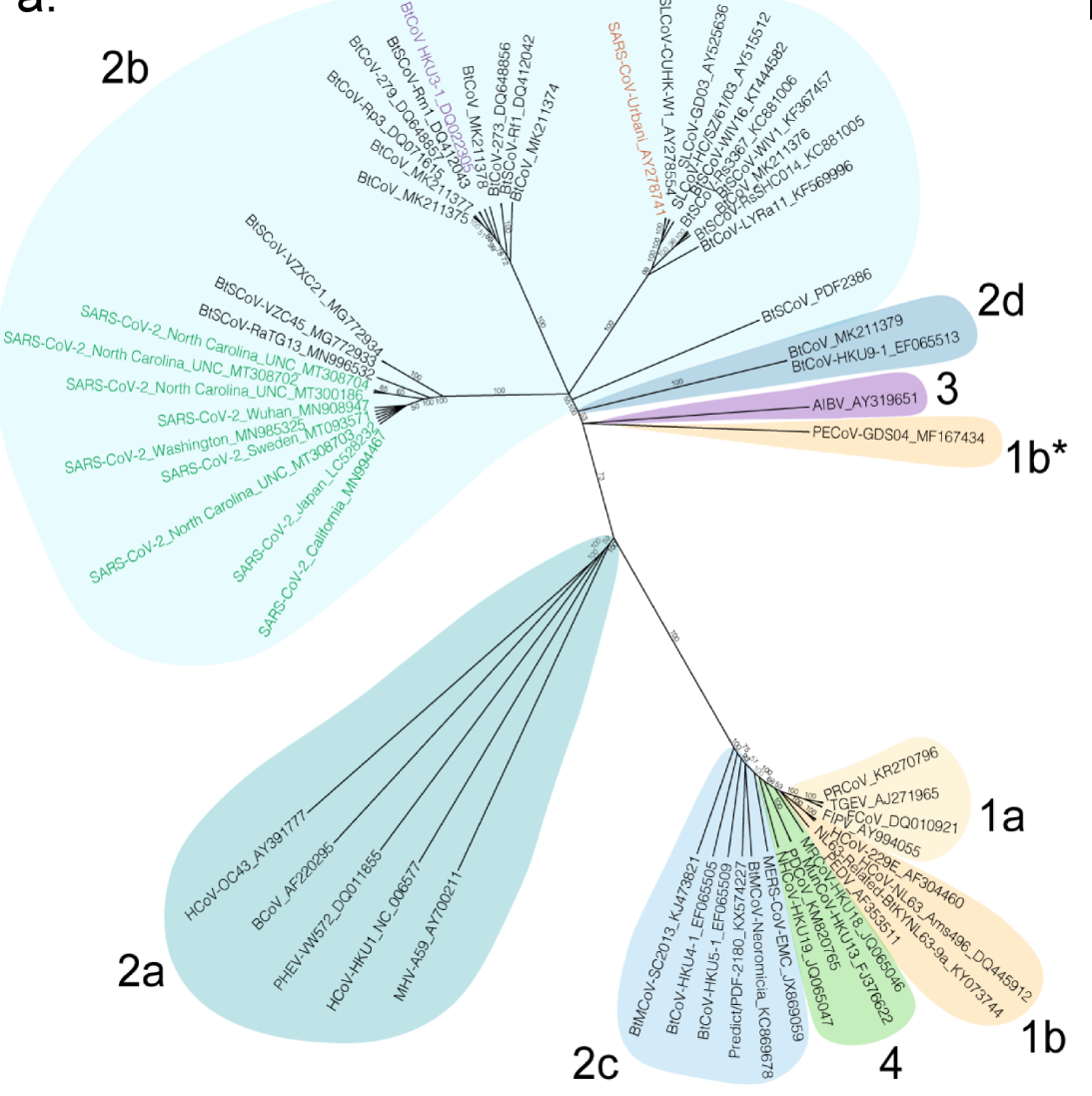

b. Virus

\begin{tabular}{|l|}
\hline SARS-CoV \\
\hline SARS-CoV-MA15 \\
\hline BtCoV-HKU3 \\
\hline SARS-CoV-2 \\
\hline
\end{tabular}

C.

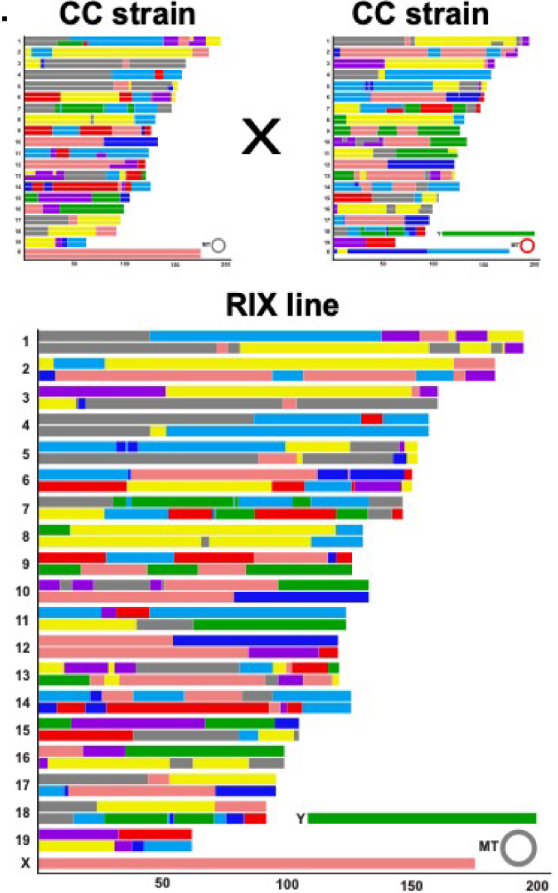

\begin{tabular}{|l|l|l|l|l|l|l|l|l|l|l|l|l|l|l|}
402 & 426 & 436 & 440 & 442 & 472 & 473 & 475 & 479 & 484 & 486 & 487 & 488 & 491 \\
\hline
\end{tabular}

\begin{tabular}{|c|c|c|c|c|c|c|c|c|c|c|c|c|c|c|}
\hline T & $R$ & $Y$ & $Y$ & Y & L & N & Y & N & Y & T & T & G & Y \\
\hline
\end{tabular}

\begin{tabular}{|l|l|l|l|l|l|l|l|l|l|l|l|l|l}
\hline T & $R$ & $H$ & $Y$ & Y & L & N & Y & N & Y & T & T & G & Y \\
\hline
\end{tabular}

\begin{tabular}{l|l|l|l|l|l|l|l|l|l|l|l|l|l} 
T & A & - & Y & S & - & - & - & S & N & N & V & P & Y \\
\hline
\end{tabular}

\begin{tabular}{l|l|l|l|l|l|l|l|l|l|l|l|l|l}
\hline T & N & Y & Y & L & F & N & Y & Q & Q & T & N & G & Y \\
\hline
\end{tabular}

d.

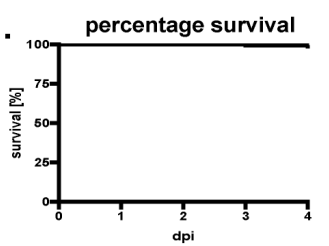

d4pi weight loss
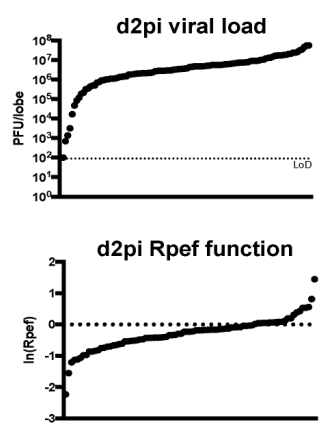

d32pi total lgG

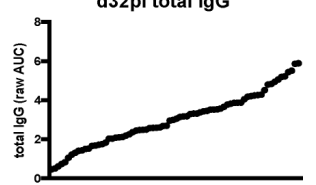

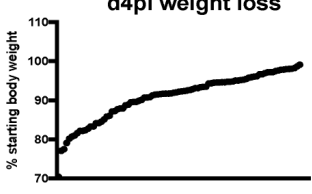

d2pi PenH function

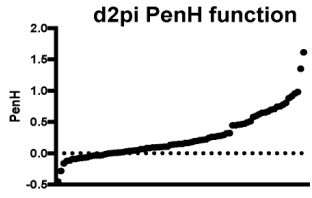

d4pi CD8+ DCs

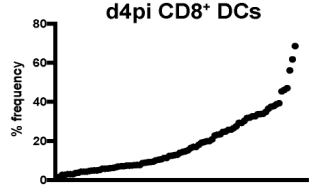

d32pi total $\lg G 1$

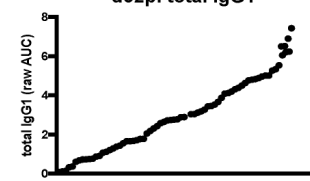




\section{Figure 2}

a. $\frac{\text { HrS13 }}{2 \text { 2dpi SARS-MA Titer (PFU/lobe) }} \quad$ HrS11
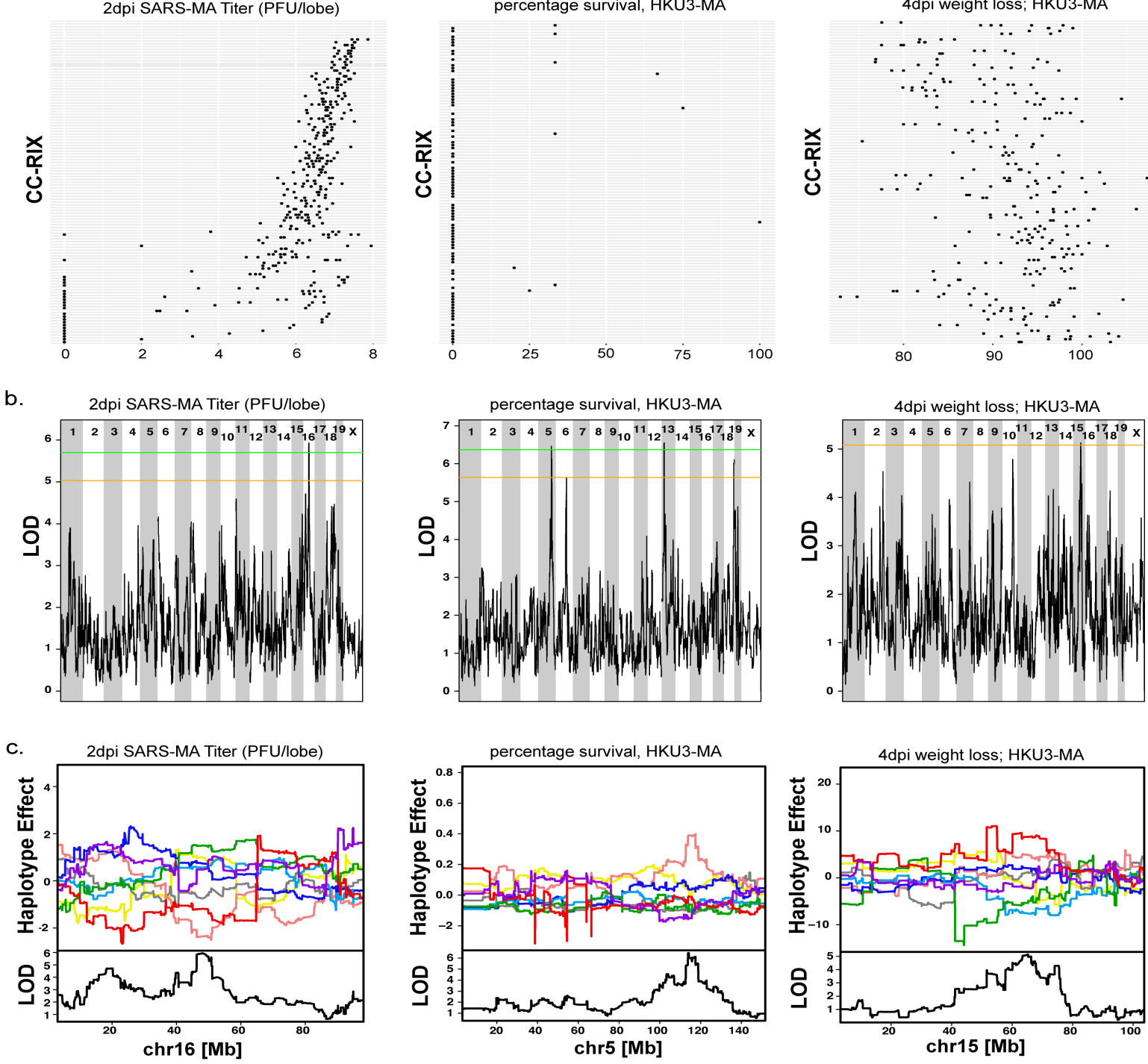

Color Legend

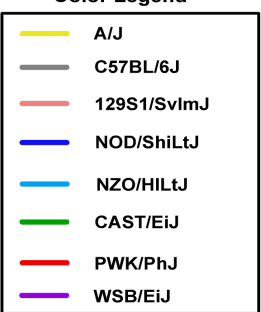
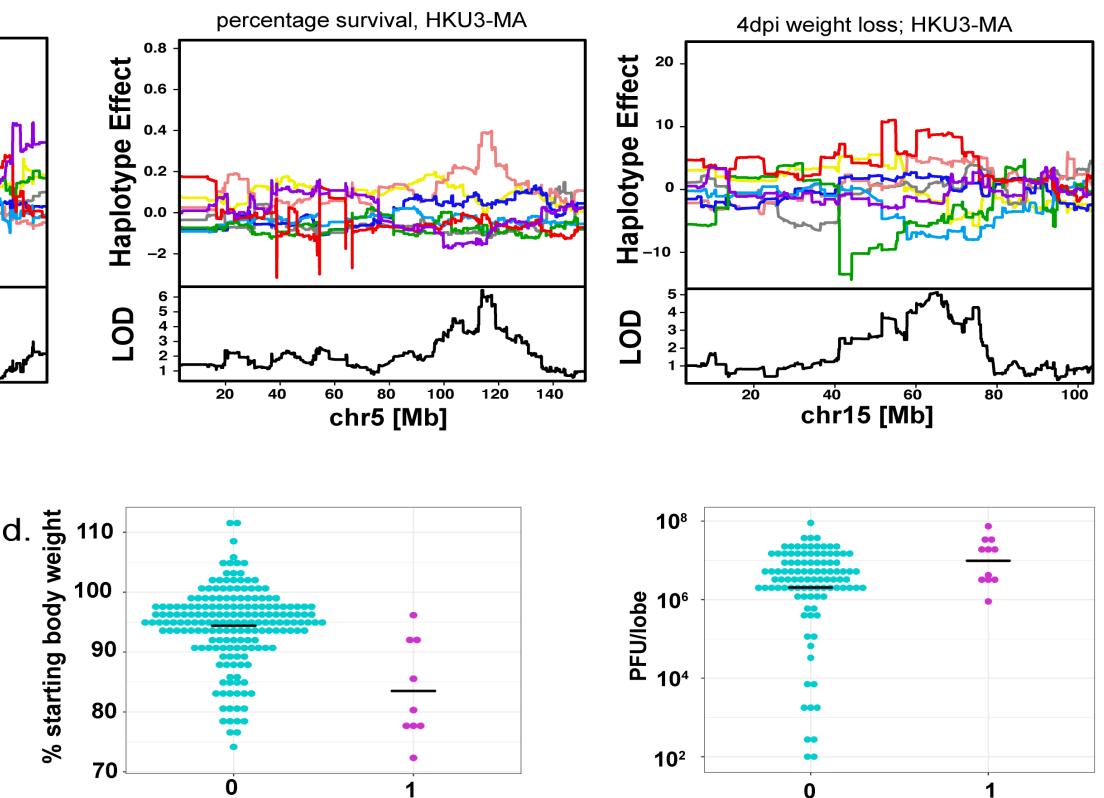

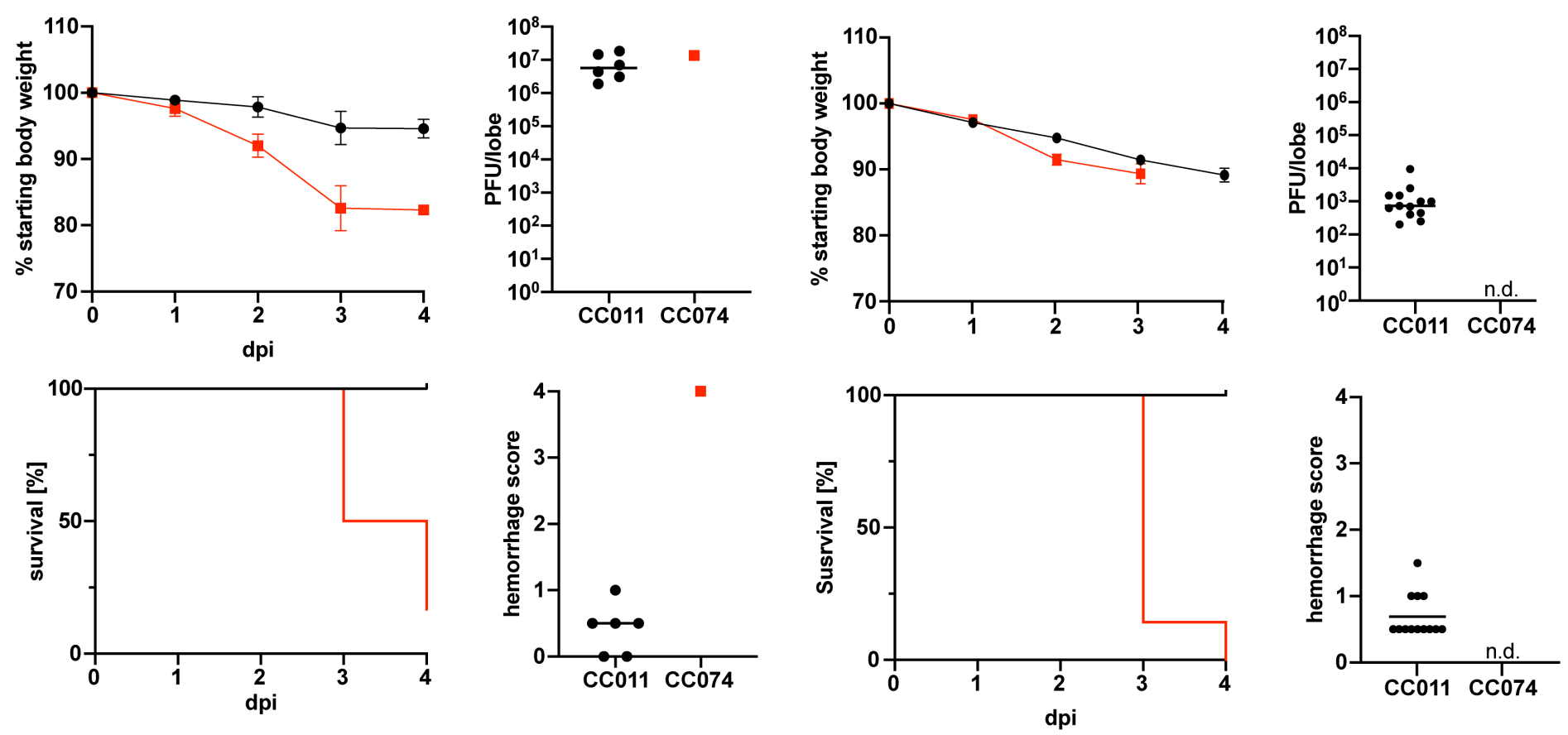

b.
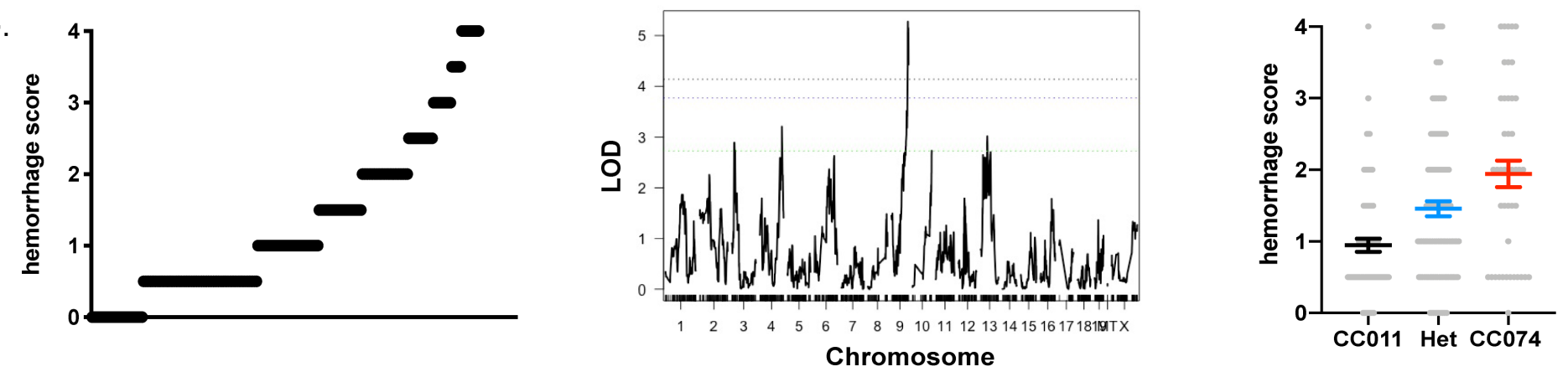

C.

QTL phenotype

circulating immune cells

lung function

histology

weight loss

mortality

Ipeaks

HrS26- d4pi neutrophils (peripheral)

HrS26- d4pi lymphocytes (peripheral)

HrS26- d2pi PenH

HrS26- d4pi lung hemorrhage

HrS26- overall mortality

HrS30- d4pi mortality

HrS30- d4pi weight loss

chr'9 
Figure 4 a.
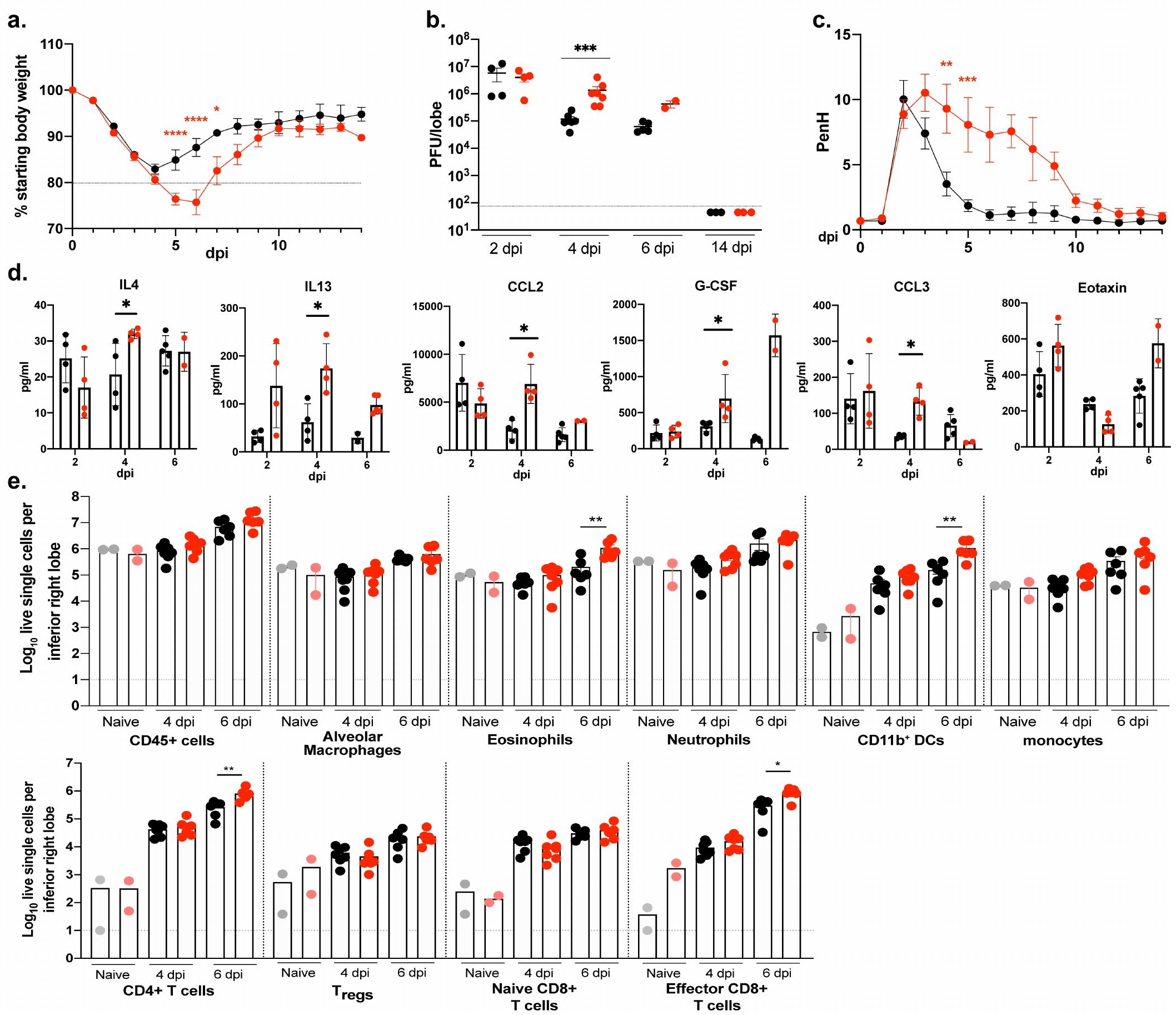
Figure 5

a. HrS23: $\mathrm{CC003 \times CC053-F2}$

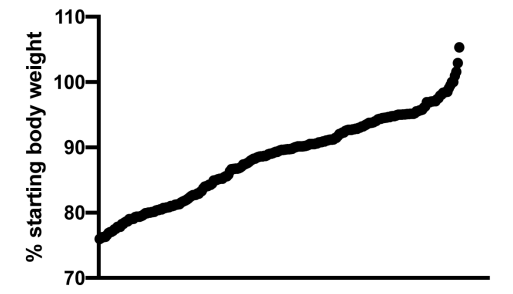

b. HrS23: $\mathrm{CC} 003 \times \mathrm{Cc} 053-\mathrm{F} 2$
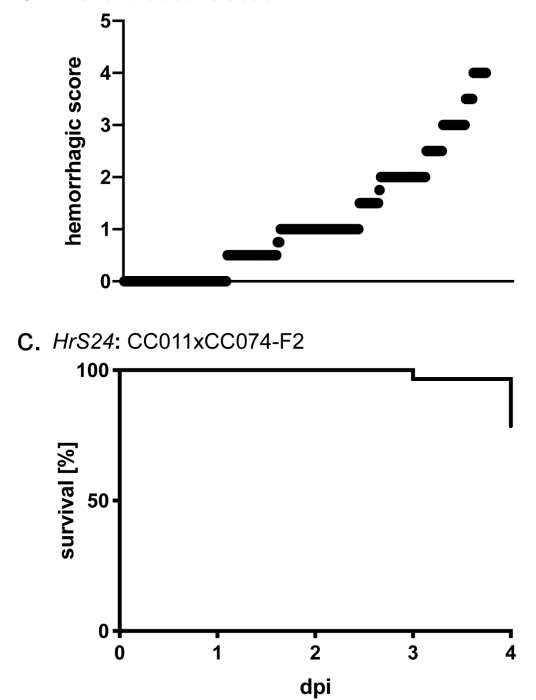

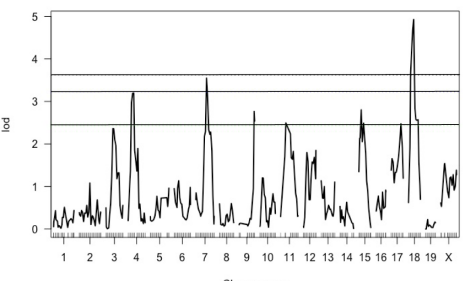

Chromosome
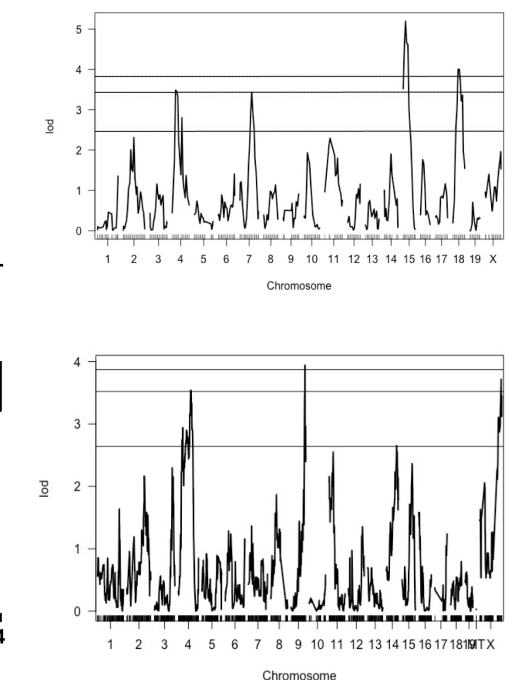

d. $\square$ C57BL/6 $\square$ TRIM14 ${ }^{\Delta 47 / \Delta 47}$
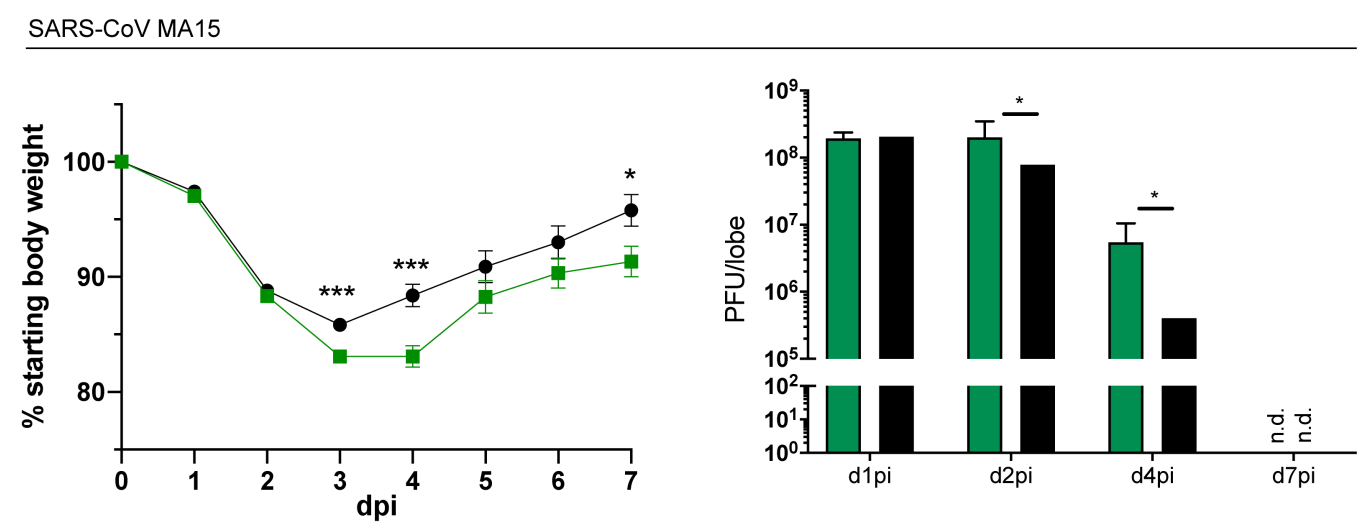

SARS-CoV-2 MA10

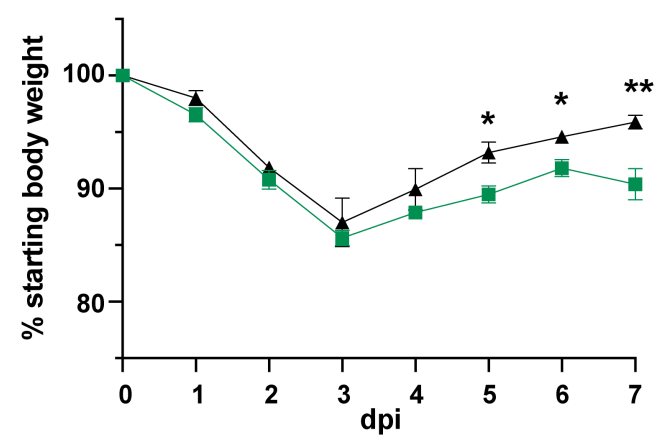




\section{Figure 6}

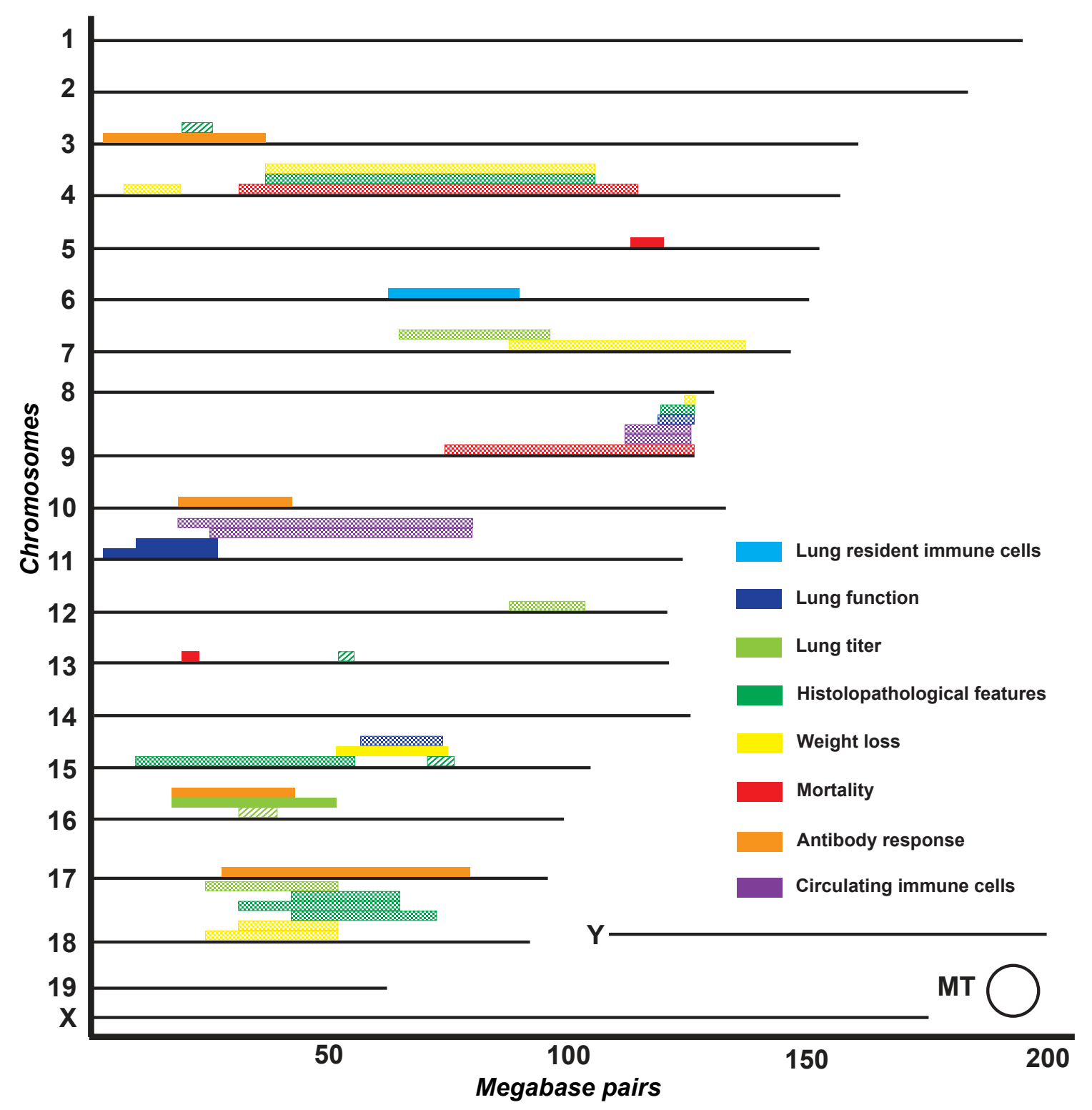




\section{Table 1}

\begin{tabular}{|c|c|c|c|c|c|}
\hline QTL ID & Population & $\begin{array}{c}\text { Chromosome } \\
{[\mathrm{Mb}]}\end{array}$ & $\begin{array}{l}\text { Phenotype(s) } \\
\text { (days post } \\
\text { infection- dpi) }\end{array}$ & Haplotype(s) $^{+}$ & $\begin{array}{c}\text { phenotypic } \\
\text { variation } \\
{[\%]}\end{array}$ \\
\hline \multicolumn{6}{|c|}{ Pre-CC } \\
\hline HrS1 & Pre-CC & chr3:18.3-26.8 & $\begin{array}{l}\text { Perivascular } \\
\text { cuffing (4dpi) }\end{array}$ & $\mathrm{BH} / \mathrm{ACDEFG}$ & $26 \%$ \\
\hline HrS2 & Pre-CC & chr16:31.5-36.7 & Viral titer (4dpi) & G/ABCDEFH & $22 \%$ \\
\hline HrS3 & Pre-CC & chr15:72.2-76.0 & $\begin{array}{l}\text { Eosinophilia } \\
\text { (4dpi) }\end{array}$ & A/BCDEFGH & $26 \%$ \\
\hline HrS4 & Pre-CC & chr13:52.7-54.9 & $\begin{array}{l}\text { Perivascular } \\
\text { cuffing (4dpi) }\end{array}$ & F/ABCDEGH & $21 \%$ \\
\hline \multicolumn{6}{|c|}{ CC-RIX } \\
\hline HrS10 & CC-RIX & chr13: 20.3- 23.7 & $\begin{array}{l}\text { HKU3-MA } \\
\text { mortality }\end{array}$ & FG/ABCDEH & $\mathrm{OR}^{*} 6.13$ \\
\hline HrS11 & CC-RIX & chr5: 112.9-118.8 & $\begin{array}{l}\text { HKU3-MA } \\
\text { mortality }\end{array}$ & C/ABDEFGH & $\mathrm{OR}^{*} 10.45$ \\
\hline HrS12 & CC-RIX & chr15: 51.5- 75.6 & $\begin{array}{l}\text { HKU3-MA weight } \\
\text { loss (4dpi) }\end{array}$ & G/ABCDEFH & $3.4 \%$ \\
\hline HrS13 & CC-RIX & chr16:18-51.1 & $\begin{array}{l}\text { SARS viral titer } \\
\text { (2dpi) }\end{array}$ & C/G/ABDEFH & $15.94 \%$ \\
\hline HrS14 & CC-RIX & chr11: $9.05-28.66$ & PenH (2dpi) & D/CEH/ABFG & $11.65 \%$ \\
\hline HrS15 & CC-RIX & chr11: 3.26- 28 & Rpef (4dpi) & ABCEFGH/D & $6.48 \%$ \\
\hline HrS17 & CC-RIX & $\begin{array}{l}\text { chr17: } 27.4-78.3 \\
\text { chr17: } 27.4-78.3\end{array}$ & $\begin{array}{l}\text { IgG1 (N) (32dpi) } \\
\text { Total IgG (N) } \\
\text { (32dpi) }\end{array}$ & A/BCDEFH/G & $\begin{array}{l}19.97 \% \\
18.53 \%\end{array}$ \\
\hline HrS18 & CC-RIX & chr16: 18.1- 43.8 & $\begin{array}{l}\text { Total lgG (N) } \\
\text { (32dpi) }\end{array}$ & ABCDEFH/G & $22.75 \%$ \\
\hline HrS19 & CC-RIX & chr10: 17.4- 130.5 & $\begin{array}{l}\text { Total IgG (N) } \\
\text { (32dpi) }\end{array}$ & ABCDEH/FG & $10.71 \%$ \\
\hline HrS2O & CC-RIX & chr3: $3.1-35.4$ & $\begin{array}{l}\text { Total IgG (N) } \\
\text { (32dpi) }\end{array}$ & G/ABCDEFH & $10.83 \%$ \\
\hline HrS22 & CC-RIX & chr6:67.2- 85 & $\begin{array}{l}\text { CD8 }{ }^{+} \text {DCs [\%] } \\
\text { (4dpi) }\end{array}$ & F/ABCDEGH & $8.17 \%$ \\
\hline \multicolumn{6}{|c|}{ CC011XCC074-F2 } \\
\hline $\mathrm{HrS24}$ & $\begin{array}{l}\text { CC011xCC074 } \\
-F 2\end{array}$ & chr4:32.95-114.54 & Mortality & $\begin{array}{l}\text { CC011: G,H,C } \\
\text { CC074:A/H, } \\
\text { A/D, D, E, F }\end{array}$ & $\mathrm{OR}^{*} 4.34$ \\
\hline HrS25 & $\begin{array}{l}\text { CC011xCC074 } \\
-F 2\end{array}$ & chr4: 6.38-17.97 & $\begin{array}{l}\text { Weight loss in } \\
\text { males (4dpi) }\end{array}$ & $\begin{array}{l}\text { CC011: G, B } \\
\text { CC074: A }\end{array}$ & $12.57 \%$ \\
\hline HrS26 & $\begin{array}{l}\text { CC011xCC074 } \\
-\mathrm{F} 2\end{array}$ & $\begin{array}{l}\text { chr9:74.94-124.06 } \\
\text { chr9:117.38- } \\
124.07 \\
\text { chr9:116.24- } \\
124.07\end{array}$ & $\begin{array}{l}\text { Mortality } \\
\text { Hemorrhage } \\
\text { (4dpi) } \\
\text { PenH (2dpi) }\end{array}$ & $\begin{array}{l}\text { CC011: B, G } \\
\text { CC074: A }\end{array}$ & $\begin{array}{l}\mathrm{OR}^{*} 3.15 \\
10.24 \% \\
7.76 \% \\
11.8 \%\end{array}$ \\
\hline
\end{tabular}




\begin{tabular}{|c|c|c|c|c|c|}
\hline & & $\begin{array}{l}\text { chr9:111.54- } \\
122.63 \\
\text { chr9:111.54- } \\
122.63\end{array}$ & $\begin{array}{l}\text { Periph. } \\
\text { neutrophils (4dpi) } \\
\text { Periph. } \\
\text { lymphocytes } \\
\text { (4dpi) }\end{array}$ & & $12.39 \%$ \\
\hline HrS27 & $\begin{array}{l}\text { CC011xCC074 } \\
-\mathrm{F} 2\end{array}$ & $\begin{array}{l}\text { chr11:26.44-80.76 } \\
\text { chr11:17.89-80.76 }\end{array}$ & $\begin{array}{l}\text { Periph. } \\
\text { neutrophils (4dpi) } \\
\text { periph. } \\
\text { lymphocytes } \\
\text { (4dpi) }\end{array}$ & $\begin{array}{l}\text { CC011: H, B, H } \\
\text { CC074: C }\end{array}$ & $\begin{array}{l}5.5 \% \\
5.6 \%\end{array}$ \\
\hline HrS28 & $\begin{array}{l}\text { CC011xCC074 } \\
-\mathrm{F} 2\end{array}$ & chr15:58.66-74.04 & PenH (2dpi) & $\begin{array}{l}\text { CC011: H } \\
\text { CC074: B }\end{array}$ & $8.48 \%$ \\
\hline \multicolumn{6}{|c|}{ CC003xCC053-F2 } \\
\hline HrS5 & $\begin{array}{l}\mathrm{CC} 003 \times \mathrm{CC} 053 \\
-\mathrm{F} 2\end{array}$ & $\begin{array}{l}\text { chr18:24.76-51.25 } \\
\text { chr18:31.63-51.25 } \\
\text { chr18:42.85-73.43 } \\
\text { chr18:31.63-65.17 } \\
\text { chr18:42.85-65.17 } \\
\text { chr18:24.76-51.2 } \\
\text { chr18:24.76-51.2 }\end{array}$ & $\begin{array}{l}\text { Weight loss (3dpi) } \\
\text { Weight loss (4dpi) } \\
\text { Hemorrhage } \\
\text { (4dpi) } \\
\text { Perivascular } \\
\text { cuffing (4dpi) } \\
\text { Edema (4dpi) } \\
\text { Viral titer (4dpi) }\end{array}$ & $\begin{array}{l}\text { CC003: A, G } \\
\text { CC053: C/G, } \\
\text { G, B, B/D, B/C, } \\
D\end{array}$ & $6 \%-13.2 \%$ \\
\hline HrS6 & $\begin{array}{l}\text { CC003xCC053 } \\
-\mathrm{F} 2\end{array}$ & chr9:121.77-end & Weight loss (3dpi) & $\begin{array}{l}\text { CC003: B, B/D } \\
\text { CC053: } \mathrm{H}\end{array}$ & $7 \%$ \\
\hline HrS7 & $\begin{array}{l}\mathrm{CC} 003 \times \mathrm{CC} 053 \\
-\mathrm{F} 2\end{array}$ & $\begin{array}{l}\text { chr7:83.45-129.93 } \\
\text { chr7:69.79-96.66 }\end{array}$ & $\begin{array}{l}\text { Weight loss (4dpi) } \\
\text { Viral titer (4dpi) }\end{array}$ & $\begin{array}{l}\text { CC003: B, C, } \\
\text { B/C } \\
\text { CC053: G/H, } \\
\text { H, D }\end{array}$ & $\begin{array}{l}6.8 \% \\
14.4 \%\end{array}$ \\
\hline HrS8 & $\begin{array}{l}\text { CC003xCC053 } \\
-\mathrm{F} 2\end{array}$ & $\begin{array}{l}\text { chr12:88.54- } \\
103.21\end{array}$ & Viral titer (4dpi) & $\begin{array}{l}\text { CC003: D } \\
\text { CC053:F/H }\end{array}$ & $4.27 \%$ \\
\hline HrS9 & $\begin{array}{l}\mathrm{CC} 003 \times \mathrm{CC} 053 \\
-\mathrm{F} 2\end{array}$ & chr15:10.7-57.3 & $\begin{array}{l}\text { Hemorrhage } \\
\text { (4dpi) }\end{array}$ & $\begin{array}{l}\text { CC003: G, } \\
\text { E/G, G } \\
\text { CC053: D, C, } \\
F\end{array}$ & $9.4 \%$ \\
\hline HrS23 & $\begin{array}{l}\mathrm{CC} 003 \times \mathrm{CC} 053 \\
-\mathrm{F} 2\end{array}$ & $\begin{array}{l}\text { chr4:35.61-104.04 } \\
\text { chr4:35.61-104.04 }\end{array}$ & $\begin{array}{l}\text { Hemorrhage } \\
\text { (4dpi) } \\
\text { Weigh loss (4dpi) }\end{array}$ & $\begin{array}{l}\text { CC003: H, E, } \\
\text { G, H } \\
\text { CC053: F, G, } \\
\text { F }\end{array}$ & $\begin{array}{l}6.86 \% \\
6.22 \%\end{array}$ \\
\hline \multicolumn{6}{|c|}{$\begin{array}{l}\text { "QTL influencing mortality calculate an Odds Ratio (OR) for disease rather than a \% of variation } \\
\text { explained. For both the CC-RIX and the CC-F2, Odds Ratios for specific loci are calculated with a full } \\
\text { model of all mortality loci, to better estimate their independent effects. } \\
{ }^{+} \text {Haplotype effects are described for each QTL. For the Pre-CC and CC-RIX, the haplotypes are } \\
\text { separated based on the allele effect splits between founder haplotypes }(A=A / J, B=C 57 B L / 6 J \text {, } \\
C=129 S 1 / S v I m J, D=N O D / S h I L t J, E=N Z O / H i L t J, F=C A S T / E i J, G=P W K / P h J, H=W S B / E i J) \text { In CC-F2 } \\
\text { crosses, the haplotypes listed are those present in the given parent strains from the proximal to distal } \\
\text { ends of each region }\end{array}$} \\
\hline
\end{tabular}


Figure S1

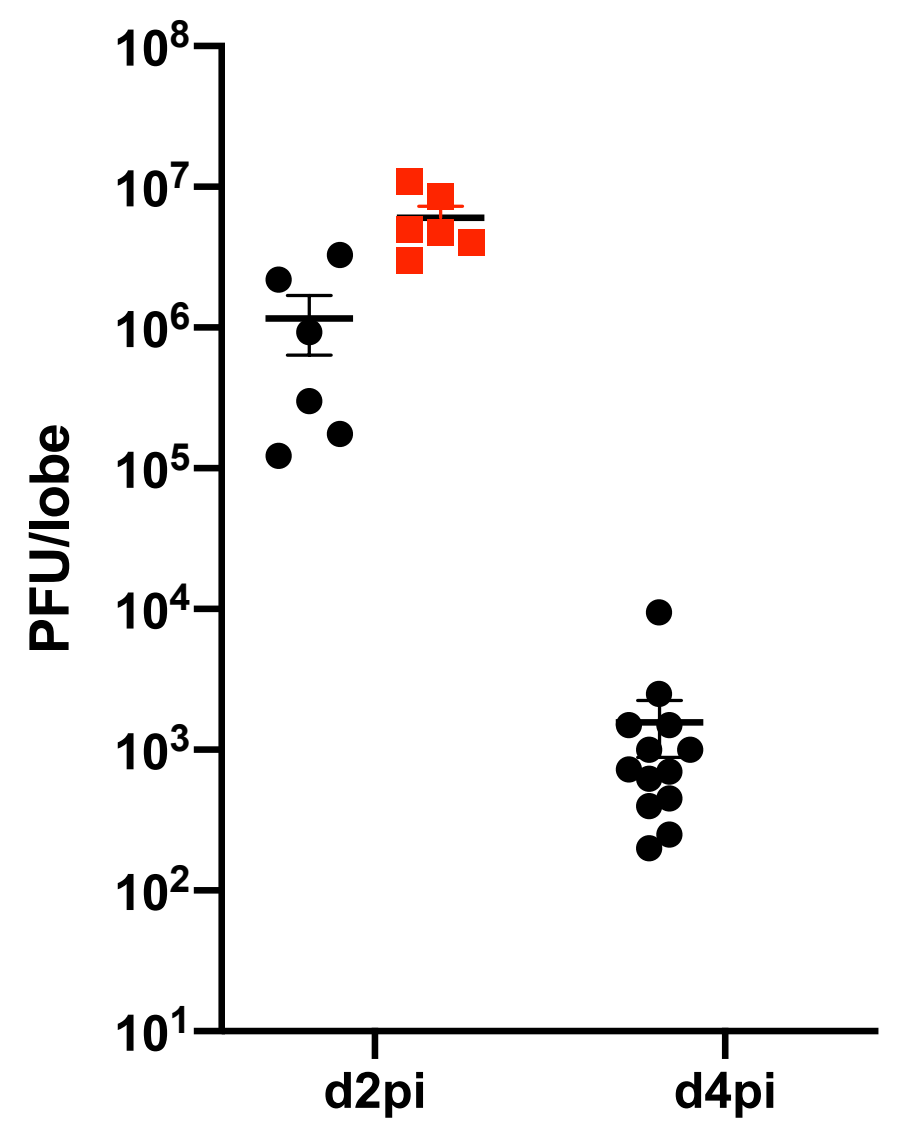

- CC011

- CC074 
Figure S2

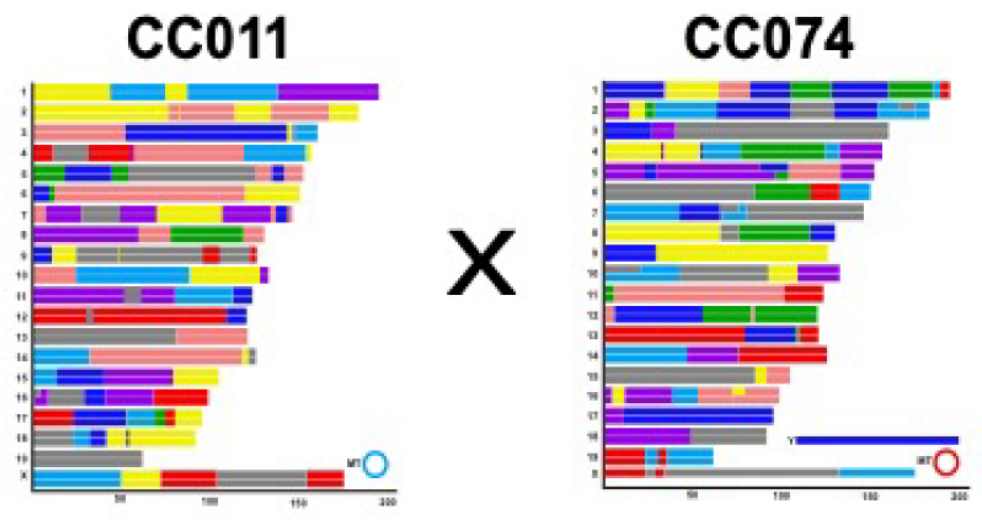

F1 generation
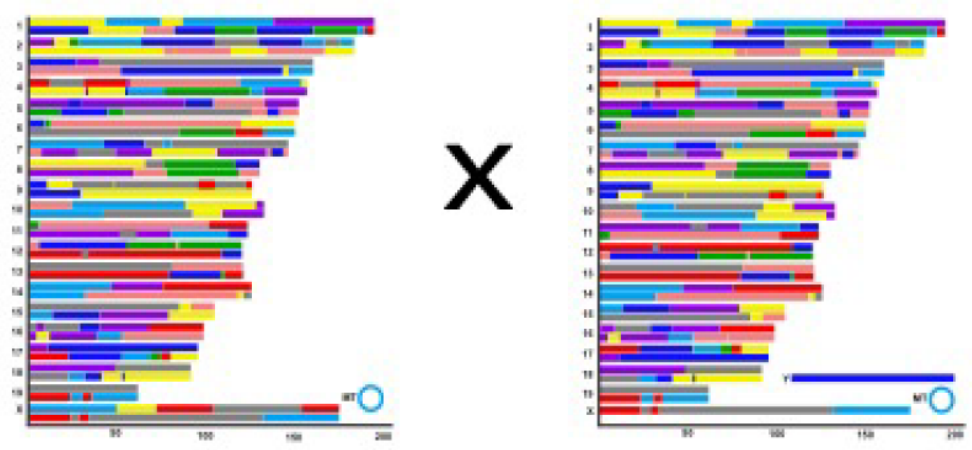

F2 generation

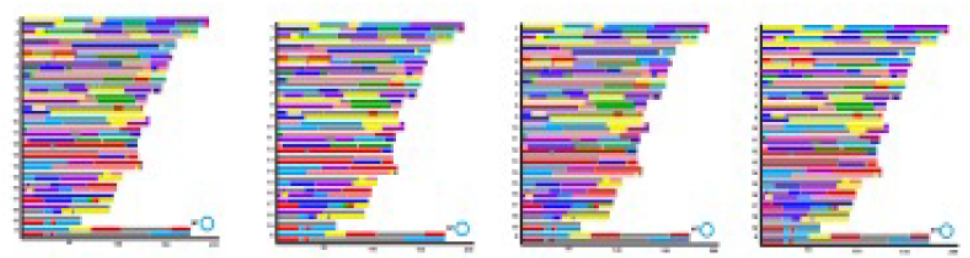


Figure S3

a.

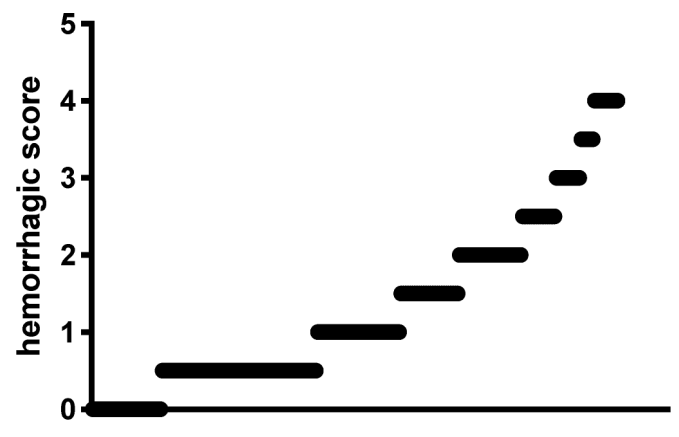

d.

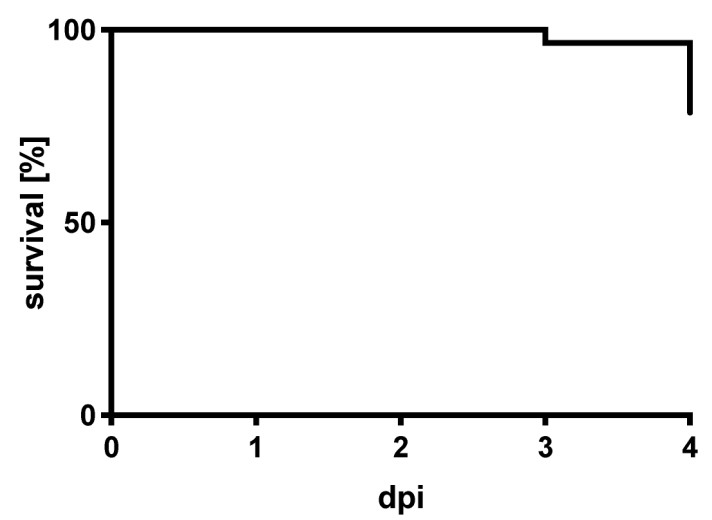

g.

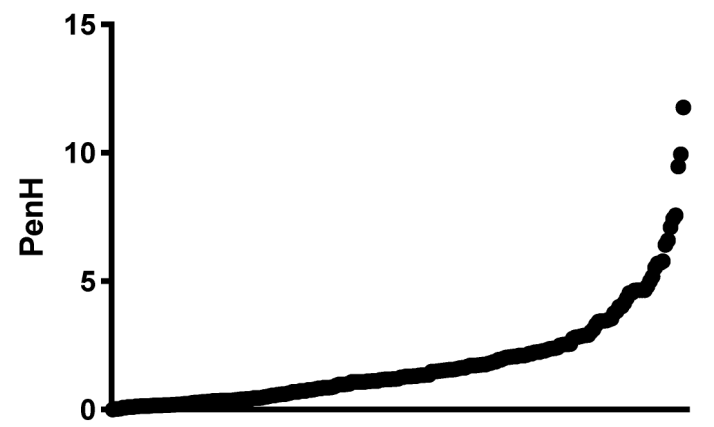

b.

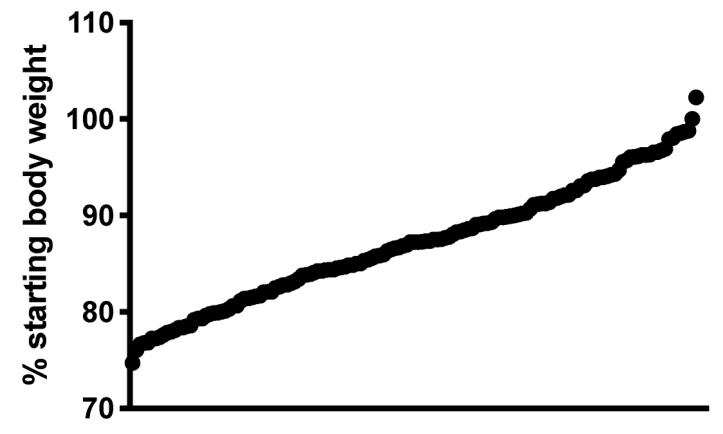

e.

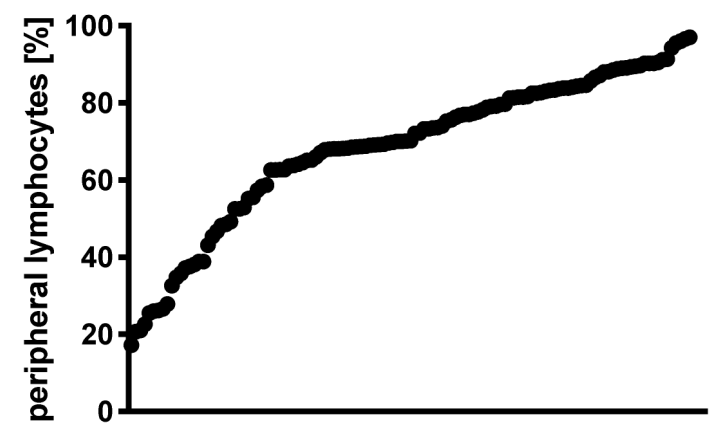

c.

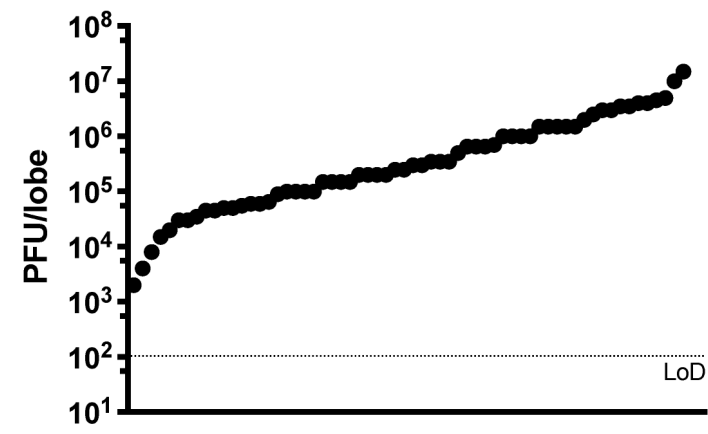

f.

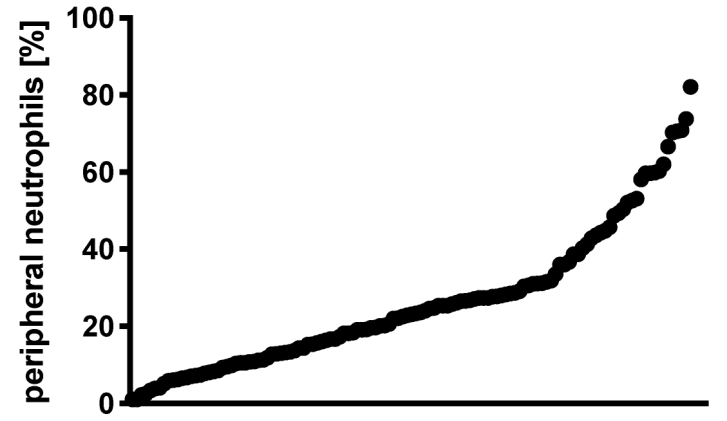


Figure S4
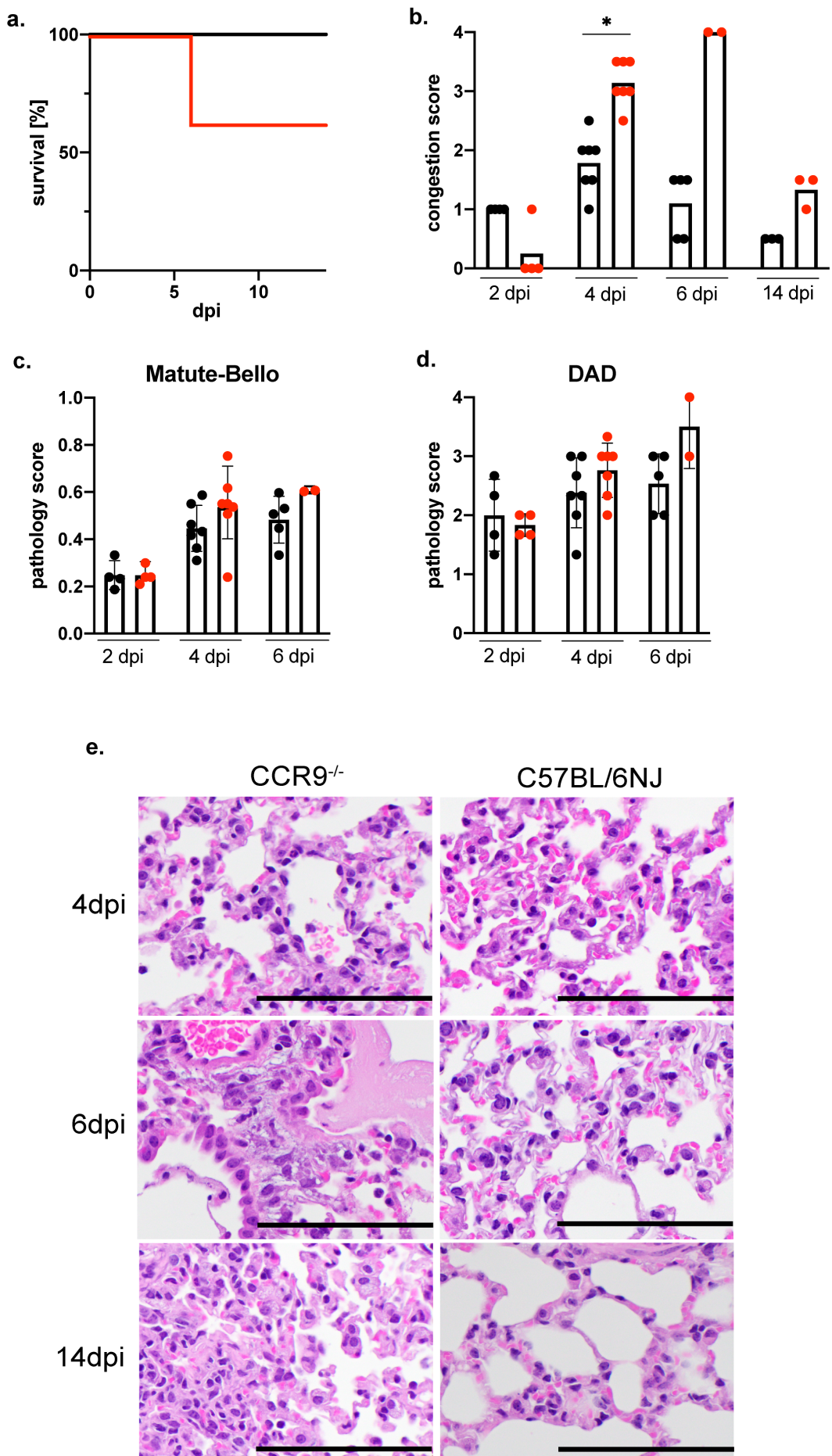
Figure S6

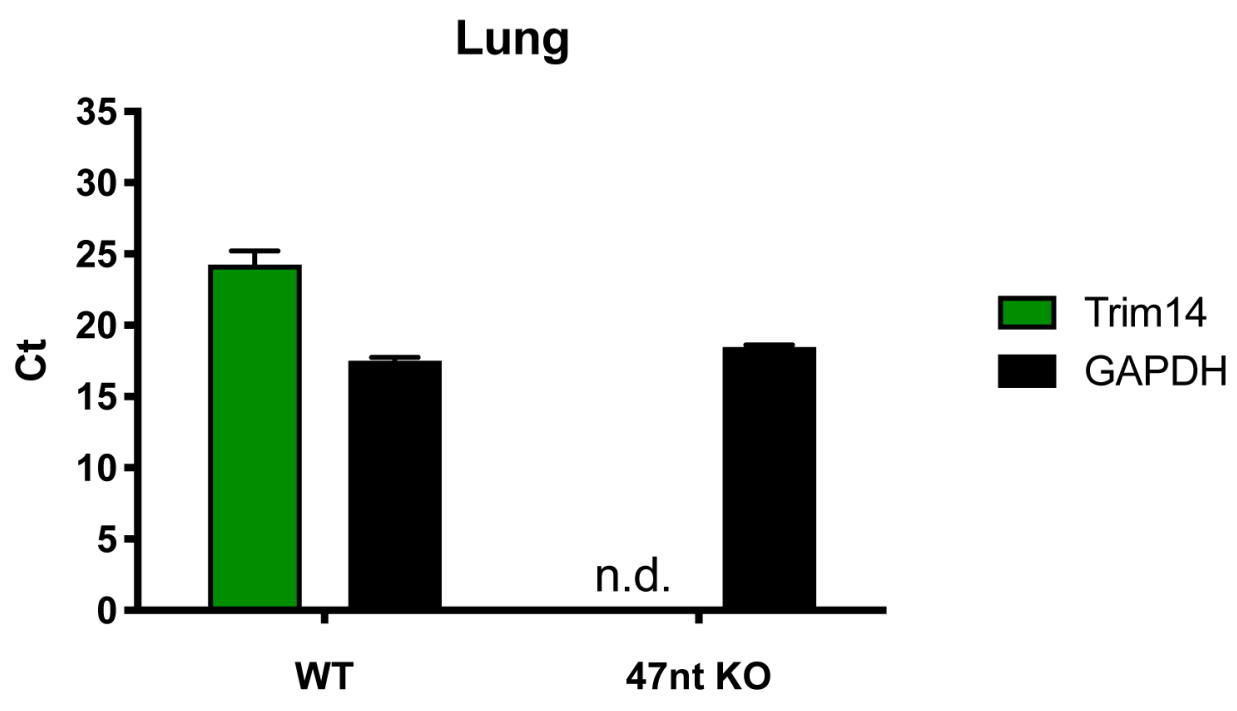




\section{Figure S7}

T Cell Gating Strategy
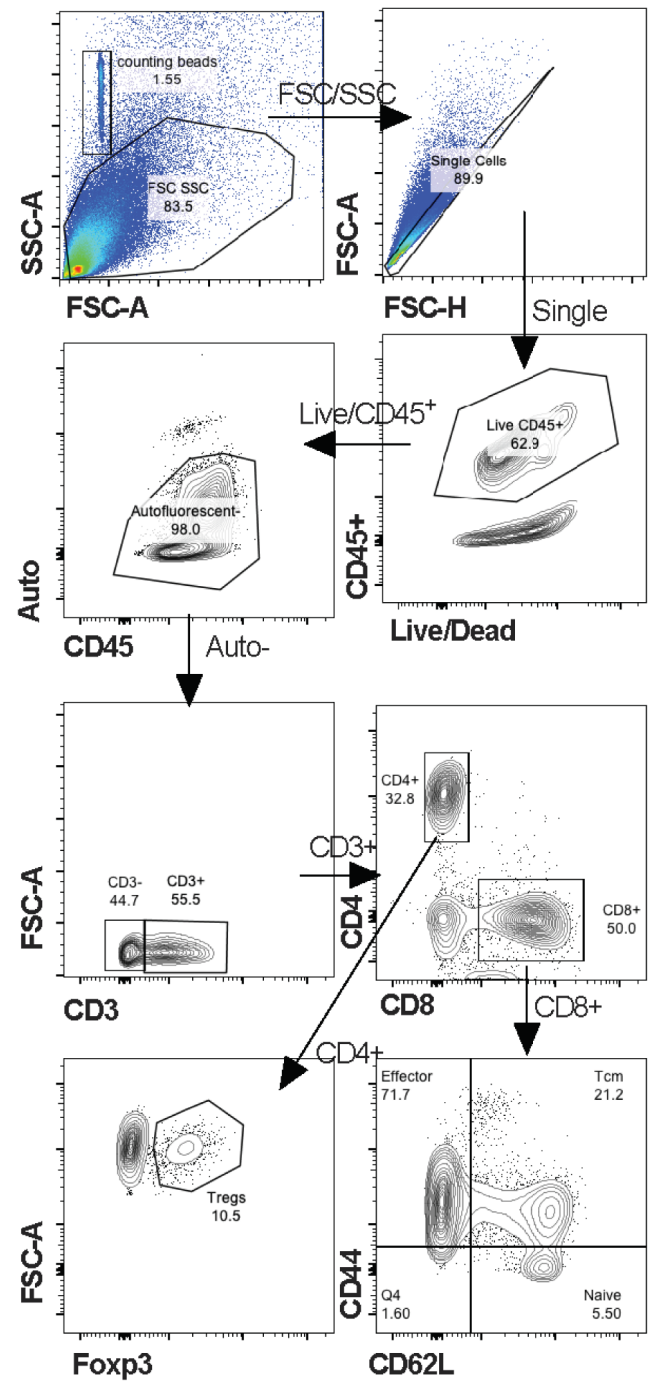

Myeloid Cell Gating Strategy
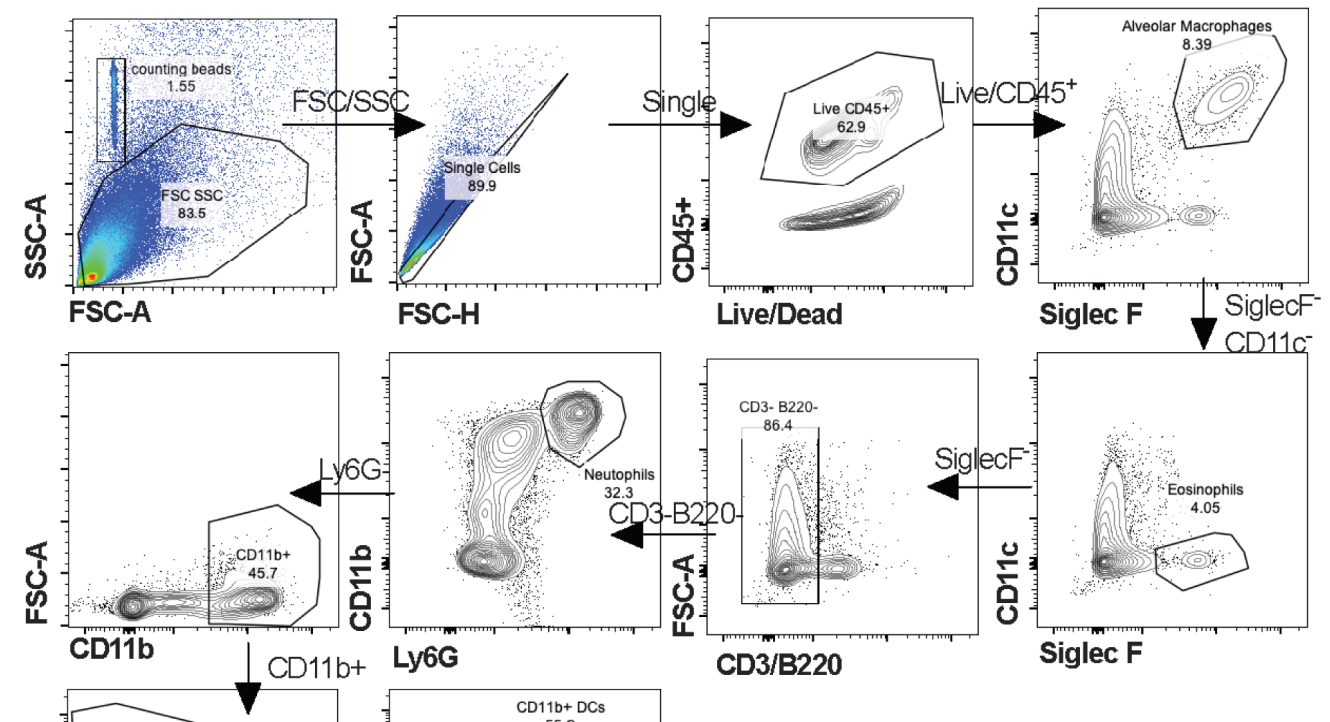
Table S1

\begin{tabular}{|c|c|c|c|}
\hline QTL ID & $\begin{array}{c}\text { Chromosome } \\
{[\mathrm{Mb}]}\end{array}$ & $\begin{array}{c}\text { Phenotype(s) } \\
\text { (days post infection } \\
- \text { dpi) }\end{array}$ & Haplotype(s) $^{+}$ \\
\hline \multicolumn{4}{|c|}{ CC011xCC074-F2 suggestive loci } \\
\hline HrS29 & $\begin{array}{l}\text { chr3: } 7.2- \\
159.1\end{array}$ & $\begin{array}{l}\text { Mortality (4dpi) } \\
\text { Hemorrhage (4dpi) }\end{array}$ & $\begin{array}{l}\text { CC011: C, C/D, D, A, D, E } \\
\text { CC074: D, H, B, B/F, B/D, B }\end{array}$ \\
\hline HrS30 & $\begin{array}{l}\text { chr9: } 28.2- \\
124.1\end{array}$ & $\begin{array}{l}\text { Weight loss (4dpi) } \\
\text { Mortality (4dpi) } \\
\text { Weight loss females } \\
\text { (4dpi) }\end{array}$ & $\begin{array}{l}\text { CC011: B, G, B } \\
\text { CC074: A }\end{array}$ \\
\hline HrS31 & $\begin{array}{l}\text { chr13: 31.9- } \\
90.3\end{array}$ & $\begin{array}{l}\text { Hemorrhage (4dpi) } \\
\text { Periph. monocytes } \\
\text { (4dpi) }\end{array}$ & $\begin{array}{l}\text { CC011: B, C } \\
\text { CC074: G, D }\end{array}$ \\
\hline HrS32 & $\begin{array}{l}\text { chr14: 33.8- } \\
114.8\end{array}$ & PenH (2dpi) & $\begin{array}{l}\text { CC011: C } \\
\text { CC074: E, H, G }\end{array}$ \\
\hline HrS33 & chrX: 6.2- 93.9 & $\begin{array}{l}\text { Rpef (2dpi) } \\
\text { Periph. neutrophils } \\
\text { (2dpi) }\end{array}$ & $\begin{array}{l}\text { CC011: E, C/E, E, A, G } \\
\text { CC074: G, B }\end{array}$ \\
\hline \multicolumn{4}{|c|}{ CC003xCC053-F2 suggestive loci } \\
\hline HrS34 & chr3: $58-148.4$ & Weight loss (3dpi) & $\begin{array}{l}\text { CC003: B, G, H, G } \\
\text { CC053: C/D, C, D, A, E }\end{array}$ \\
\hline HrS35 & chr17: 6- 79 & Weight loss (3dpi) & $\begin{array}{l}\text { CC003: C/D, C, C/D, D, B } \\
\text { CC053: G, E, G, H, A, G, H }\end{array}$ \\
\hline HrS36 & $\begin{array}{l}\text { chr9: } 3.6 \mathrm{Mb}- \\
74.9 \mathrm{Mb}\end{array}$ & $\begin{array}{l}\text { Weight loss (4dpi), } \\
\text { Titer (4dpi), } \\
\text { Edema (4dpi) }\end{array}$ & $\begin{array}{l}\text { CC003: C, B } \\
\text { CC053: B, E }\end{array}$ \\
\hline HrS37 & $\begin{array}{l}\text { chr10: } 5.9 \mathrm{Mb}- \\
86.3 \mathrm{Mb}\end{array}$ & Titer (4dpi) & $\begin{array}{l}\text { CC003: C, H } \\
\text { CC053: H, B, A }\end{array}$ \\
\hline HrS38 & $\begin{array}{l}\text { chrX: 9.4Mb- } \\
\text { 169.5Mb }\end{array}$ & Titer (4dpi) & $\begin{array}{l}\text { CC003: C, G, C/G, C/E, C, E } \\
\text { CC053: A, C, B, F }\end{array}$ \\
\hline HrS39 & $\begin{array}{l}\text { chr2: } 4.3 \mathrm{Mb}- \\
181.8 \mathrm{Mb}\end{array}$ & mortality (4dpi) & $\begin{array}{l}\text { CC003: } D, B, E \\
\text { CC053: } B, E, G, E, A, C, F\end{array}$ \\
\hline HrS41 & $\begin{array}{l}\text { chr6: } 34.2 \mathrm{Mb}- \\
114 \mathrm{Mb}\end{array}$ & $\begin{array}{l}\text { Interstitial septum } \\
\text { (4dpi), } \\
\text { Airspace inflammation } \\
\text { (dpi4), } \\
\text { Eosinophils (4dpi) }\end{array}$ & $\begin{array}{l}\text { CC003: B, E } \\
\text { CC053: } G, A, A / D, G\end{array}$ \\
\hline HrS42 & $\begin{array}{l}\text { chr11: } 37 \mathrm{Mb}- \\
121.6 \mathrm{Mb}\end{array}$ & Edema (4dpi) & $\begin{array}{l}\text { CC003: A/C, B/C, A/C, B/H, B, } \\
\text { E, H, C, E } \\
\text { CC053: B }\end{array}$ \\
\hline \multicolumn{4}{|c|}{$\begin{array}{l}{ }^{+} \text {Haplotype effects are described for each QTL. For F2 crosses, the haplotypes listed are } \\
\text { those present in the given parent strains from the proximal to distal ends of each region } \\
(A=A / J, B=C 57 B L / 6 J, C=129 S 1 / S v I m J, D=N O D / S h I L t J, E=N Z O / H i L t J, F=C A S T / E i J, \\
G=P W K / P h J, H=W S B / E i J) . \text { In }\end{array}$} \\
\hline
\end{tabular}

